\title{
An adaptive fuzzy approach for modelling visual texture properties
}

\author{
Jesús Chamorro-Martínez and Pedro Manuel Martínez-Jiménez \\ Department of Computer Science and Artificial Intelligence \\ University of Granada, Spain \\ José Manuel Soto-Hidalgo \\ Department of Computer Architecture, Electronics \\ and Electronic Technology, University of Córdoba, Spain \\ Belén Prados-Suárez \\ Department of Software Engineering, University of Granada, Spain
}

This is the peer reviewed version of the following article: Jesús ChamorroMartínez, Pedro Manuel Martínez-Jiménez, José Manuel Soto-Hidalgo, Belén Prados-Suárez: An adaptive fuzzy approach for modeling visual texture properties. Fuzzy Sets and Systems 286: 86-113 (2016), which has been published in final form at https://doi.org/10.1016/j.fss.2015.09.008. This article may be used for non-commercial purposes in accordance with Creative Commons Attribution Non-Commercial No Derivatives License (CC-BY-NC-ND license): https://creativecommons.org/licenses/by-nc-nd/4.0/legalcode.

\begin{abstract}
The analysis of the perceptual properties of texture plays a fundamental role in tasks like semantic description of images, content-based image retrieval using linguistic queries, or expert systems design based on low level visual features. The presence of these properties in images is very difficult to characterize due to their imprecision, and, moreover, because their perception may change depending on the user or the image context. In this paper, texture properties are modelled by means of an adaptive fuzzy approach that takes into account the subjectivity of the human perception. For this purpose, a methodology in two phases has been proposed. First, non-adaptive fuzzy models, that represent the average human perception about the presence of the texture properties, are obtained. For this modelling, we propose to learn a relationship between representative measures of the properties and the assessments given by human subjects. In a second phase, the obtained fuzzy sets are adapted in order to model the particular perception of the properties that an user may have, as well as the changes in perception influenced by the image context. For this purpose, the membership functions are automatically
\end{abstract}


transformed on the basic of the information given by the user or extracted from the image context, respectively.

Keywords: fuzzy sets; image processing; texture modelling; human perception; adaptive models

\section{Introduction}

Color, texture, and shape are typically the three most used features for object recognition and image interpretation. Color and shape represent clear concepts for humans, and their importance is widely known in computer vision. Texture, however, is more imprecise and abstract but an equally important feature. In spite of its importance, there is not an accurate definition for the concept of texture, but some widespread intuitive ideas. Texture is described by some authors as local changes in the intensity patterns or gray tones, which is used in opposition to the homogeneity idea [1]. Other authors consider texture as a set of basic items called texels (or texture primitives), arranged in a certain way. However, for humans, the most common way to describe texture is by using vague textural properties, like coarseness, directionality, contrast, line-likeness or regularity [2,3], that are a more natural way to represent our perception about texture primitives. Coarseness is related to the spatial size of texels, directionality reflects whether they have a dominant orientation, contrast is related to their distinguishability, line-likeness reflects whether they have straight shapes, and regularity refers to the variation of their placement. From all of them, and according to the psychological experiments performed by Tamura et al. in [3], coarseness, contrast and directionality are considered the three most important texture properties, playing a fundamental role in human visual interpretation $[4,5,6]$. In this paper, we will focus our study on these properties.

Computational models with the ability of providing a perceptual texture characterization on the basis of these properties can be very useful in tasks where some interaction with subjects is needed. For example, they can be applied in fields such as semantic description of images [7, 8, 9], obtaining texture descriptions that are directly interpretable by humans, or in content-based image retrieval systems $[10,11,4]$, where linguistic queries related to the degree to which texture properties are present can be employed ${ }^{1}$. In addition, this perceptual characterization of texture can be also applied in expert systems, where the information provided by the expert is related to the presence of the texture properties. For example, suppose a medical expert that, according to his/her experience, concludes that the regions with high fineness presence and high contrast degree in microscopic images are indicative of a certain disease. Models that are able to provide a textural description in a similar way as humans would can be employed to automatically identify these areas in the images.

\footnotetext{
${ }^{1}$ Notice that the aim in this case is not to retrieve images with a similar texture as a whole (like in classical texture analysis approaches in the literature), but to retrieve images with a similar degree of presence in certain, required texture properties.
} 


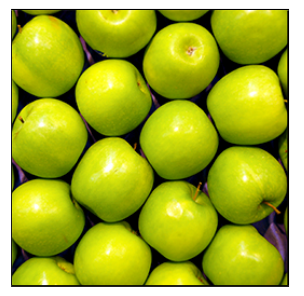

(a)

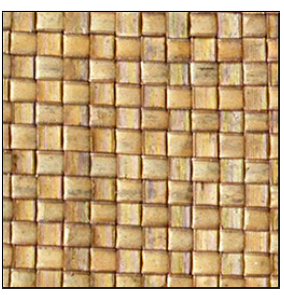

(b)

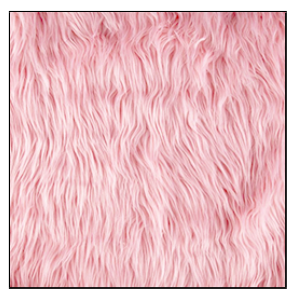

(c)

Figure 1: Examples showing the imprecision associated to the properties.

However, there are two main problems related to the modelling of the perceptual properties of texture. The first one is the imprecision associated to them. This imprecision must be understood in the sense that, except in extreme cases, we cannot set a precise threshold between textures that strictly accomplish a property and textures that do not, but the fulfillment of the property is gradual in nature. For example, we can reasonably say that the texture shown in Figure 1(a) is coarse and contrasted, and that the texture shown in Figure 1(c) is not, as they represent potential extreme cases for both properties. However, the fulfillment of these properties is not so clear for the texture shown in Figure 1 (b).

This way, it is natural for humans to give assessments about the degree to which these perceptual properties are present. For example, if a subject is asked about the degree of the coarseness presence in the images of Figure 1, this subject would probably say that the texture shown in Figure 1(a) is very coarse, the texture shown in Figure 1(b) has an intermediate coarseness degree, or the texture shown in Figure 1(c) is very fine. Likewise, if the subject is asked about the degree of the contrast presence, these textures may be perceived with a high degree, low degree and very low degree of this property, respectively.

The second main drawback related to the modelling of texture properties is the subjectivity associated to their perception. On the one hand, the perception of a texture property may change depending on the user. For example, although we have considered that the texture shown in Figure 1(a) is very coarse, another user may consider that this texture is not so coarse. On the other hand, the image context may affect in the global perception of the texture properties. An example of this fact can be shown in Figure 2. The images in figures 2(a) and 2(b) are very similar, but in the last one a new texture has been added. The presence of this texture, that is much coarser than the others, can inhibit the rest of textures, and they may be perceived as finer than in Figure $2(\mathrm{a})^{2}$. Thus, in addition to the imprecision associated to the textural properties, it should be taken into account that the greater or lesser fulfillment of these properties in texture may be different for different users, and it may also depend on the

\footnotetext{
${ }^{2}$ This effect is more noticeable if the images are observed separately.
} 


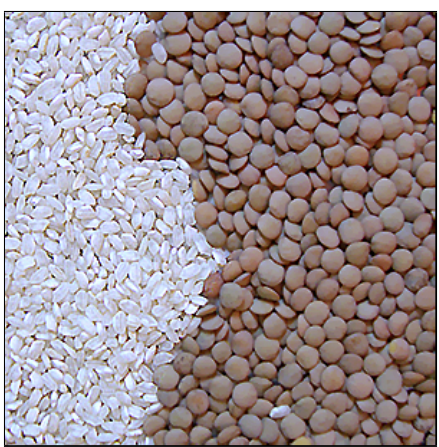

(a)

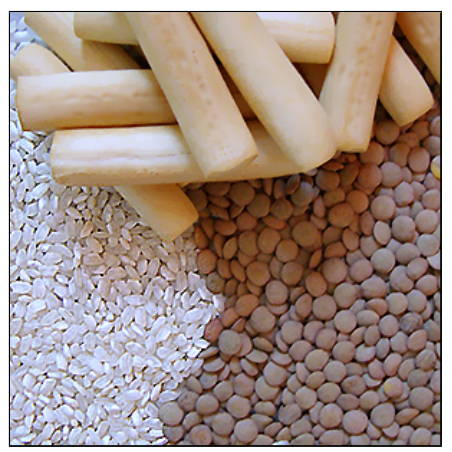

(b)

Figure 2: Example showing the influence of the image context in the perception of the fineness property.

image context.

In this paper, an adaptive fuzzy approach is proposed in order to address the problems commented above, that, to the best of our knowledge, have not been solved in the literature. Our aim is to obtain models that are able to represent the degree to which the textural properties are present in a similar way as humans would, taking into account the imprecision associated to them, as well as the subjectivity of the human perception. The proposed methodology has two phases. In the first one, non-adaptive fuzzy models are obtained, that can be used if additional information (the particular perception of the user or the image context) is not available. In our approach, we propose to model the properties of coarseness, contrast and directionality (although other properties can be dealt with using our methodology) by means of fuzzy sets defined on the domain of representative measures of these properties. In order to obtain the membership function associated to each fuzzy set, a functional relationship between the computational values given by the measures and the human perception of the property is learned. To get information about the human perception, a set of images covering different degrees to which the properties are present are used to collect, by means of polls, human assessments from a set of subjects. This way, the obtained non-adaptive fuzzy sets will represent the average perception about the presence of the texture properties. In addition, goodness measures are proposed in order to identify the most appropriate models to represent the properties of coarseness, contrast and directionality.

In the second phase of our approach, a methodology for adapting these generic models to the particular perception of a new user or the image context is proposed. For this purpose, the membership functions associated to these fuzzy sets are automatically adapted by means of a functional transformation on the basis of the new perception. In the case of the adaptation to an user's profile, a set of texture images representing the particular perception of this user 
are employed in the transformation process. This way, the degree of presence given by the adapted model will match what the user would expect. In the case of the adaptation to the image context, the textures present in the image are analized in order to obtain information about the current inhibition. This information is used in the adaptation process, obtaining models that represent the degree to which the property is present influenced by the context.

The rest of the paper is organized as follows. Section 2 describes the related work in the literature. In section 3 a general overview of our methodology is presented, introducing some basic concepts and the notation used in the paper. The computational measures used as reference set are summarized in section 4 . The method used to obtain the fuzzy sets that define the non-adaptive models is described in section 5, while section 6 introduces the procedure employed to adapt the membership functions to new perceptions of the texture properties. In section 7 a comparative study of these models with the state of the art and some results obtained by applying them are shown, while section 8 summarizes the main conclusions and future works.

\section{Related work}

The majority of the image analysis techniques in computer vision try to model texture by means of feature vectors (that usually have very large dimensions) which have no direct relationship with the different perceptual properties. These approaches, that are based on multiresolution analysis and scale-space theory, such as Gabor functions [12, 13, 14] or Wavelets [15, 16, 17], do not provide a textural representation interpretable by humans. On the contrary, they are intended for comparing different textures on the basis of the similarity between their feature vectors, which is a very important but completely different problem.

Although, we can find in the literature some techniques that propose a texture characterization based on its perceptual properties, most of these approaches are crisp proposals $[18,19,3,20]$ which do not take into account the imprecision related to texture. To address this problem, some techniques arise from the fuzzy set field, and more specifically from the content-based image retrieval area [10, 4, 21, 22, 23]. In these proposals, a mapping from low-level statistical features (the crisp measures mentioned above) to high level textural concepts is performed by defining membership functions for each textural feature.

However, all these fuzzy approaches have four main drawbacks, that, to the best of our knowledge, have not been addressed in the literature. First of all, in these proposals membership functions with a fixed form (usually triangles or trapezoids) are used, which prevents an accurate modelling of the textural concepts. Moreover, linear functions are used for transitions, although it is known [24] that computational measures do not have a linear variation with the degree to which the property is present. The second drawback is that the parameters that define these membership functions are adjusted manually or by using a fuzzy clustering, but without considering the relationship between the measure 
values and the human perception of the property. This implies that the obtained membership degrees do not necessarily match what a human would expect. The third drawback is that they do not propose a global modelling of the textural concept, but a fuzzy partition providing a set of linguistic terms associated to this concept. This type of solution is unsuitable for some classical tasks, like pattern recognition, because a single degree to which the textural property is present cannot be obtained, but one membership degree for each linguistic term in the partition. And finally, the fourth drawback of these fuzzy approaches is that they do not take into account the subjectivity associated to the perception of the texture properties (the changes in the perception depending on the user and the image context), as it has been commented in the previous section.

The adaptive fuzzy approach proposed in this paper allows to solve all these problems. First, parametric polynomial functions are used for fuzzy sets, which allows to introduce more degrees of freedom than membership functions with a fixed form; second, the parameters of the membership functions are obtained by considering the human perception of the corresponding property, which allow to obtain membership degrees that match what a human would expect; third, each texture property is modelled by means of a unique fuzzy set, i.e. we propose to model each textural concept as a whole instead of using a fuzzy partition; and fourth, the proposed methodology allows to adapt the obtained fuzzy models to the particular perception of an user and to the changes in perception influenced by the image context.

In some of our previous works $[25,24,26]$, preliminary studies about the modelling of the fineness property have been presented in order to address the first three problems commented above. In this paper we have extended these studies in two ways. First, an adaptive methodology has been proposed in order to solve the fourth problem, i.e. to take into account the subjectivity of the perception in the modelling of texture properties; and second, we have incorporated the fuzzy modelling of the other two properties that, as discussed in the previous section, have more influence in the human perception of visual texture: contrast and directionality. The modelling of each of these properties poses different problems and requires to perform a set of non-trivial tasks to solve them, as we will show throughout this paper.

\section{Preliminaries and notations}

As mentioned in the above sections, the objective of this paper is to assess the presence of texture properties in the image by means of fuzzy sets. From now on, let $\mathcal{P}=\left\{\right.$ fineness $^{3}$, contrast, directionality $\}$ be the set ofthe perceptual properties of texture that will be modelled in this paper and let $\mathcal{F}^{p}=\left\{F_{1}^{p}, \ldots, F_{K_{p}}^{p}\right\}$ be a set of representative computational measures of the property $p \in \mathcal{P}$. In our approach, we propose to model the presence of a texture property $p \in \mathcal{P}$ as

\footnotetext{
${ }^{3}$ Let us remark that "coarseness" and "fineness" are opposite but related textural concepts. The advantage of modelling the concept of fineness is that the maximum presence of this property in the image is delimited by the size of the pixels.
} 
a fuzzy set $\hat{\mathcal{T}}_{k}^{p}$ defined on the domain of a measure $F_{k}^{p}$, where the membership function ${ }^{4}$ of this fuzzy set will be defined as

$$
\hat{\mathcal{T}}_{k}^{p}: \mathbb{R} \rightarrow[0,1]
$$

For this modelling, two questions need to be addressed: (i) what reference set should be used for the fuzzy set, and (ii) how to obtain the related membership function. Regarding the reference set, as mentioned above, we will define the fuzzy set on the domain of a given computational measure $F_{k}^{p} \in \mathcal{F}^{p}$. The measures analyzed in this paper, corresponding to fineness, contrast and directionality properties, are summarized in section 4. All of them are automatically computed from the texture images.

Regarding the membership function, we propose to obtain it by using a perceptually-based approach that relates the computational measures with the human perception of the property. This approach is divided into two phases, that are illustrated in the flowchart shown in Figure 3. In the first one, an initial fuzzy set $\mathcal{T}_{k}^{p}$ (that has been called non-adaptive model) is obtained in order to model the average perception about the property $p \in \mathcal{P}$. This fuzzy set is obtained by learning a relationship between each measure (the reference set) and the human perception of the property extracted from a poll. The membership function associated to this fuzzy set is defined as

$$
\mathcal{T}_{k}^{p}: \mathbb{R} \rightarrow[0,1]
$$

The methodology used to obtain $\mathcal{T}_{k}^{p}$ will be described in detail in section 5. If we do not want to take into account a different perception of the texture property, the non-adaptive model $\mathcal{T}_{k}^{p}$ can be used directly, i.e. $\hat{\mathcal{T}}_{k}^{p}=\mathcal{T}_{k}^{p}$. However, if we want to consider the particular perception of a new user or the image context, the second phase of this approach needs to be applied.

In the second phase of our approach, the fuzzy set $\hat{\mathcal{T}}_{k}^{p}$ is obtained by adapting the generic model $\mathcal{T}_{k}^{p}$ in order to take into account the subjectivity in the perception of the property $p \in \mathcal{P}$. This way, the adapted model $\hat{\mathcal{T}}_{k}^{p}$ is adjusted to the particular perception of a new user by means of the information obtained from a set of texture images provided by the user. Moreover, the change in the perception due to the image context is taken into account by analyzing the textures present in the image. The adaptation process will be described in detail in section 6 .

\section{Computational measures: the reference set}

There are many measures in the literature that, given an image, capture the presence of a textural property in the sense that the greater the value given by the measure, the greater (lower) the presence of the property. In this section, the measures used in our study, corresponding to fineness, contrast and directionality properties, are summarized.

\footnotetext{
${ }^{4}$ To simplify the notation, as it is usual in the scope of fuzzy sets, we will use the same notation $\hat{\mathcal{T}}_{k}^{p}$ for the fuzzy set and for the membership function that defines it.
} 


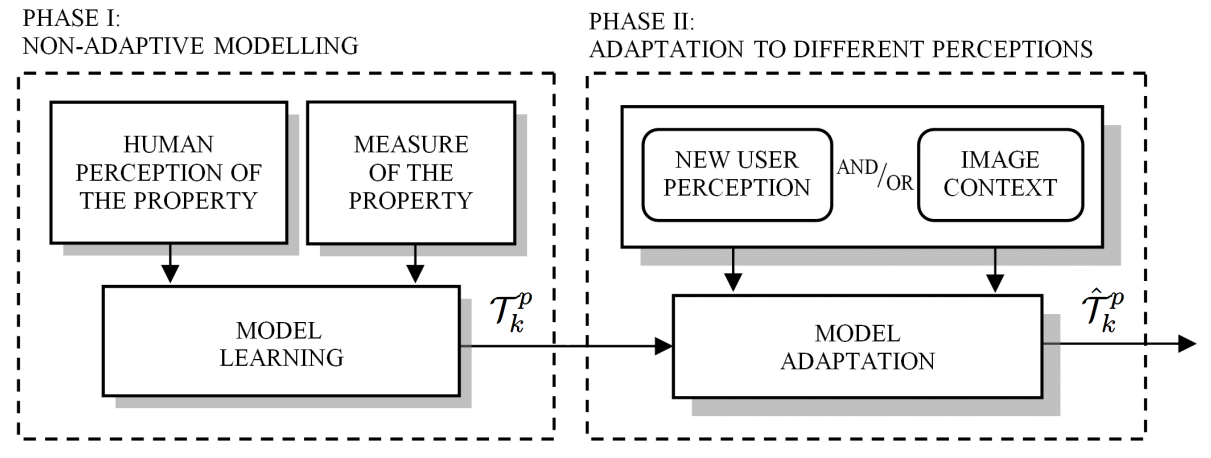

Figure 3: Flowchart illustrating the proposed methodology.

\subsection{Fineness measures}

Among all the perceptual texture properties, the coarseness-fineness is the most popular one, being considered as the most fundamental feature in texture analysis by some authors [4]. In fact, the presence of fineness is usually associated to the presence of texture (from this point of view, texture is defined as local variations against the idea of homogeneity). In this sense, a fine texture contains small texture primitives with large gray tone differences between neighbor pixels (e.g. the first image of Figure 4(a)), whereas a coarse texture corresponds to larger primitives formed by several pixels (e.g. the last image of Figure 4(a)).

In this paper, we have initially considered the 17 fineness measures analyzed in our previous work [24]. These measures can be classified into 3 groups according to the strategy used to quantify the coarseness of the texture image. The first group includes those measures that try to estimate directly the size of the texels by analyzing the pixels of the image. In this group we can find the measure defined by Abbadeni et al. in [20], the measure proposed by Tamura et al. in [3], the Edge Density (ED measure), that is calculated as the percentage of pixels which are an edge in the image, and the Fractal Dimension (FD measure) defined by Mandelbrot in [27], that is estimated by following the blanket method introduced by Peleg in [28].

The second group includes the measures obtained by applying statistics over matrices that collect information about the relationships between the gray level of each pixel and their neighbours. The measures of Haralick [18], that are based on the GLCM matrix, are placed in this group. In particular, 6 coarseness measures are obtained by applying the statistics contrast, correlation, entropy, local homogeneity, variance and uniformity over this matrix. This group also includes the measure defined by Amadasun in [19], the Short Run Emphasis (SRE measure) given by Galloway in [29], the Small Number Emphasis (SNE measure) defined by Sun et al. in [30], the Distribution of Gray Level Difference (DGD measure) proposed by Kim et al. in [31], and the measure defined by Weszka et al. in [32]. 


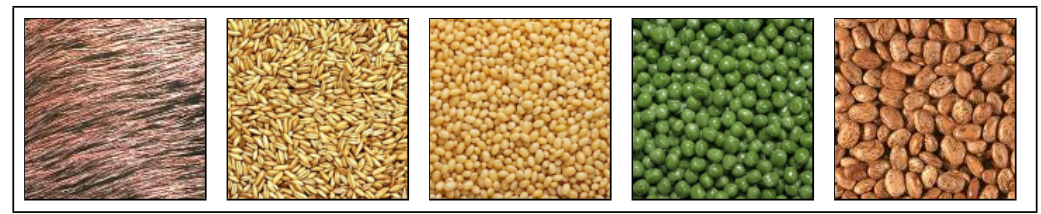

(a)

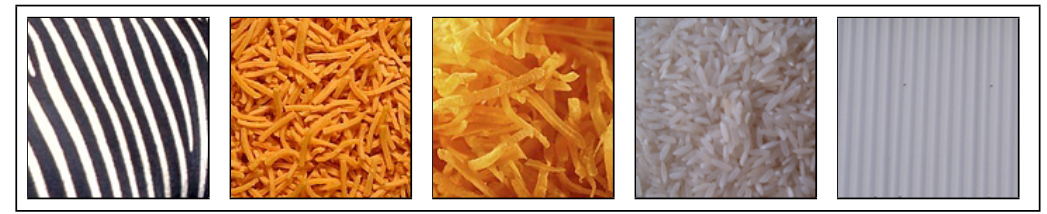

(b)

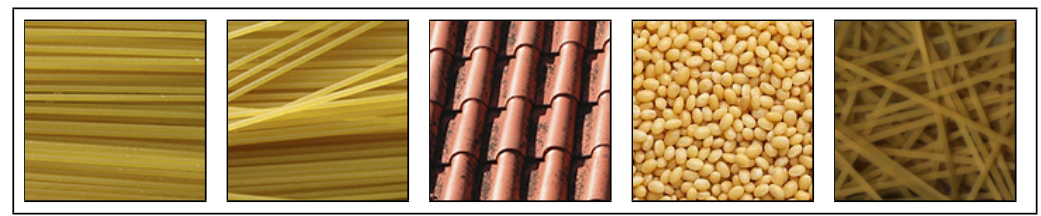

(c)

Figure 4: Examples of texture images used in the poll corresponding to the properties of fineness (a), contrast (b) and directionality (c).

The third group is composed of two measures that are based on the Fourier power spectrum of the image. The first one is the measure used by Newsam in [33], computed as the average of power spectrum over ring-shaped regions centered at the origin, and the second one is the first moment of the power spectrum (FMPS measure), obtained by computing the mean value of Fourier power spectrum of the image [34].

However, according to the study performed in [24], some of the above measures have an unsuitable behavior. The measures of Newsam, FMPS, Entropy and uniformity are size dependent, i.e. the values given by these measures are affected by the window size. In addition, the Variance measure does not provide a representative information about the perception of fineness. Thus, these five measures are rejected and they will not be taken into account in the following, focusing our study on the other 12 measures, that are listed in the first column of Table 1(a). Besides independence with respect to the image size and the ability to provide information about the fineness perception, other interesting properties for texture analysis are also fulfilled by the proposed measures. As it is shown in [24], they are robust against changes in the image characteristics, like brightness and contrast, and they are not significantly affected by the presence of noise in the image. 


\subsection{Contrast measures}

The contrast property reflects the clarity with which texture primitives are distinguishable. In this sense, a well contrasted texture contains primitives that are clearly visible and separable (e.g. the first image of Figure 4(b)), whereas a low contrasted texture has a poor distinguishability between texels (e.g. the last image of Figure 4(b)). Notice that this property refers to grayscale images, and it should not be confused with the contrast related to color images.

In this paper, we propose to use 4 of the most used contrast measures in the literature. Two of them try to estimate directly the contrast between texels by analyzing the pixels of the image. The first one is the measure defined by Tamura et al. in [3], which takes into account both the dynamic range of gray levels in the image and the kurtosis of their distribution. The second one is the contrast measure defined by Abbadeni in [20], which is based on the autocovariance function.

The other two measures are obtained by applying statistics over matrices that collect information about the relationships between the gray level of each pixel and their neighbours. The first one is the contrast statistic proposed by Haralick et al. in [18], which is obtained from GLCM matrices. The second one is the contrast measure proposed by Amadasun and King in [19], which takes into account both global statistics (as the dynamic range of gray levels in the image) and local statistics calculated from the Neighbourhood Gray-Tone Difference Matrix.

\subsection{Directionality measures}

The directionality property is related to the presence of a dominant orientation in texture primitives, and it depends on two factors: (i) the shape of texture primitives individually, and (ii) their placement rule in the image. In this sense, a directional texture contains primitives that have a dominant dimension, i.e. texels with an elongated shape, and, in addition, this primitives are arranged in the same orientation (e.g. the first image of Figure 4(c)). The directionality presence decreases as the orientation of all texels does not match, even if they have an elongated shape (e.g. the second image of Figure 4(c)), or as the shape of texels is less elongated, even if they are arranged in the same orientation (e.g. the third image of Figure 4(c)). The no directionality presence (omnidirectional texture) is associated to texture primitives that does not have a dominant dimension in their shape (e.g. the fourth image of Figure 4(c)) or their arrangement does not have a dominant orientation (e.g. the last image of Figure 4(c)).

In this paper, we will use 3 of the most known directionality measures in the literature. The first one is the directionality measure defined by Tamura et al. in [3], which is based on the computation of an histogram of local edge probabilities against their directional angle. The second one is the directionality

measure proposed by Abbadeni in [20], which is also based on the analysis of directional angles, but considering the local edges obtained from the autocovari- 
ance function, instead of the local edges of the original image. The last measure is the directionality statistic computed from the Fourier power spectrum of the image [32, 33], which analyzes the energy distribution in wedge-shaped regions of the frequency domain.

\section{Non-adaptive modelling}

In this section, the methodology used to obtain the non-adaptive models $\mathcal{T}_{k}^{p}$ will be described. As mentioned above, we propose to obtain the memberships function of these fuzzy sets by learning a relationship between the computational measures and the human perception of the corresponding property.

For this purpose, two questions need to be addressed: firstly, how to obtain the data about the "human perception" of the property and, secondly, how to fit these data with the measures in order to obtain the membership function. To get information about the human perception of a texture property $p \in \mathcal{P}$, a set of images covering different degrees to which this property is present has been gathered. These images are used to collect, by means of a poll, human assessments about the perceived presence of the property. From now on, let $\mathcal{I}^{p}=$ $\left\{I_{1}^{p}, \ldots, I_{N_{p}}^{p}\right\}$ be the set of $N_{p}$ images representing examples of the property $p \in \mathcal{P}$, and let $\Gamma^{p}=\left\{v_{1}^{p}, \ldots, v_{N_{p}}^{p}\right\}$ be the set of human assessments associated to $\mathcal{I}^{p}$. The description of the texture image set and the way to obtain $\Gamma^{p}$ are detailed in section 5.1.

To obtain the membership function $\mathcal{T}_{k}^{p}$ for a given measure $F_{k}^{p} \in \mathcal{F}^{p}$ of the texture property $p \in \mathcal{P}$, a robust fitting method will be applied in order to obtain suitable functions relating the values of the measure calculated for each image with the presence degree of the property $p$ perceived by humans. This fitting method is described in section 5.2.

\subsection{Assessment collection}

In this section, the way to obtain the set of values $\Gamma^{p}=\left\{v_{1}^{p}, \ldots, v_{N_{p}}^{p}\right\}$, that represent the presence degree of the property $p \in \mathcal{P}$ perceived by humans in the images $I_{i}^{p} \in \mathcal{I}^{p}$, will be described. For this purpose, first the image set $\mathcal{I}^{p}$ will be selected (section 5.1.1). After that, a poll for getting assessments about the perception of the property will be designed (section 5.1.2). Finally, for a given image, the assessments of the different subjects will be aggregated (5.1.3).

\subsubsection{The texture image set}

For each property $p \in \mathcal{P}$, a set $\mathcal{I}^{p}=\left\{I_{1}^{p}, \ldots, I_{N_{p}}^{p}\right\}$ of $N_{p}=80$ images representing examples of this property has been selected. Figure 4 shows some images extracted from the set $\mathcal{I}^{p}$ corresponding to the properties of fineness (Figure $4(\mathrm{a})$ ), contrast (Figure 4(b)) and directionality (Figure 4(c)). Each set has been selected satisfying the following conditions:

- It covers the different degrees to which the property is present. 
- The number of images for each degree of presence is representative enough.

- Each image shows, as far as possible, just one degree of presence of the property.

Due to the third condition, each image can be viewed as "homogeneous" with respect to the presence degree of the corresponding property, i.e., if we select two random windows (with a dimension which does not "break" the original texture primitives and structure), the perceived presence of the property will be similar for each window (and also with respect to the original image). In other words, we can see each image $I_{i}^{p} \in \mathcal{I}^{p}$ as a set of lower dimension images (sub-images) with the same degree of presence as the original one. This will be very useful for the fitting process, because we can have a larger number of fitting points without extending the number of images used in the poll.

\subsubsection{The poll}

Given the image set $\mathcal{I}^{p}$, the next step is to obtain assessments about the perception of the corresponding property $p \in \mathcal{P}$ from a set of subjects. From now on, we will denote by $\Theta_{i}^{p}=\left[o_{i, 1}^{p}, \ldots, o_{i, L}^{p}\right]$ the vector of assessments obtained from $L$ subjects for the image $I_{i}^{p}$. We considered two alternatives to get $\Theta_{i}^{p}$ :

- To ask subjects about a degree of presence between 0 and 1 for each image in the set.

- To ask subjects to assign images to classes, so that each class has associated a degree of presence. In our proposal, an example image which represents the degree of presence is associated to each class.

The first choice allows subjects to have more freedom to assess the degree to which the property is present. However, according to our own experience, it

is very difficult for a subject to provide a value between 0 and 1 that represents degree to which a certain texture concept is present (except in the case of both extremes: fulfillment of the concept -degree of 1- and unfulfillment of the concept -degree of 0 ). Thus, this alternative was discarded.

The above problem is solved by the second choice. The subject does not assess a value but classifies each image into a class, giving his opinion about the degree to which the texture property is present. From now on, let $R^{p}$ be the number of classes that have been considered in the poll for the property $p \in \mathcal{P}$. One of the classes represents the presence degree of 1 of this property. In our proposal, traditional examples used in the literature to define very fine, high contrasted and very directional textures has been considered for this class [35]. An example that represents this presence degree for each property is shown in the first image of figures 4(a), 4(b) and 4(c), respectively. Another of the classes considered in the poll represents the presence degree of 0 of the property. In this case, again, traditional examples used in the literature to define very coarse, very low contrasted and very non-directional textures has been considered for 
this class. The last image of figures 4(a) and 4(b) shows an example of this presence degree for fineness and contrast properties, respectively. In the case of directionality, the presence degree of 0 is associated to texture primitives that do not have a dominant dimension or their arrangement does not have a dominant orientation, as it is shown in the last two images of Figure 4(c).

The rest of classes represent presence degrees of the property between 0 and 1. In the case of fineness, nine classes have been employed, considering gradual variations in the size of texture primitives. Figure 4(a) shows the sample images associated to five of these classes, ordered in a decreasing presence degree of the fineness concept. In the case of contrast, five classes have been considered, taking into account gray level differences in texel edges in order to scale their contrast between the classes corresponding to degrees 1 and 0 . The five representative images used in the poll are shown in Figure 4(b). In the case of directionality, five classes have been also employed, considering gradual variations in texel orientation or/and shape, as it is shown in the second and third images of Figure 4(c). Note that, since it is more difficult for humans to discriminate between different presence degrees of contrast and directionality than between the fineness degree, the number of classes considered for these two properties is smaller.

In our approach, 20 subjects have participated in the poll. As result, a vector of 20 assessments $\Theta_{i}^{p}=\left[o_{i, 1}^{p}, \ldots, o_{i, 20}^{p}\right]$ is obtained for each image $I_{i}^{p} \in \mathcal{I}^{p}$. The degree $o_{i, j}^{p}$ associated to the assessment given by the subject $S_{j}$ to the image $I_{i}^{p}$ is computed as $o_{i, j}^{p}=\left(R^{p}-k\right) /\left(R^{p}-1\right)$, where $k \in\left\{1, \ldots, R^{p}\right\}$ is the index of the class to which the image is assigned by the subject.

\subsubsection{Assessment aggregation}

Our aim at this point is to obtain, for each image in the set $\mathcal{I}^{p}$, one assessment $v_{i}^{p}$ that summarizes the assessments $\Theta_{i}^{p}$ given by the different subjects about the presence degree of the property $p \in \mathcal{P}$. To aggregate opinions we have used an OWA operator guided by a quantifier [36]. Concretely, the quantifier "the most" has been employed, which allows to represent the opinion of the majority of the subjects. This quantifier is defined as

$$
Q(r)=\left\{\begin{array}{lr}
0 & \text { if } r<a \\
\frac{r-a}{b-a} & \text { if } a \leq r \leq b, \\
1 & \text { if } r>b
\end{array}\right.
$$

$\forall r \in[0,1]$, with $a=0.3$ and $b=0.8$. Once the quantifier $Q$ has been chosen, the weighting vector of the OWA operator can be obtained following Yager [36] as $w_{j}=Q(j / L)-Q((j-1) / L), j=1,2, \ldots, L$. According to this, for each image $I_{i}^{p} \in \mathcal{I}^{p}$, the vector $\Theta_{i}^{p}$ obtained from $L$ subjects will be aggregated into one assessment $v_{i}^{p}$ as follows:

$$
v_{i}^{p}=w_{1} \hat{o}_{i, 1}^{p}+w_{2} \hat{o}_{i, 2}^{p}+\ldots+w_{L} \hat{o}_{i, L}^{p}
$$


where $\left[\hat{o}_{i, 1}^{p}, \ldots, \hat{o}_{i, L}^{p}\right]$ is a vector obtained by ranking in nonincreasing order the values of the vector $\Theta_{i}^{p}$.

\subsection{Fitting the membership Function}

At this point, the aim is to obtain, for a given measure $F_{k}^{p} \in \mathcal{F}^{p}$ of a property $p \in \mathcal{P}$, the corresponding membership function $\mathcal{T}_{k}^{p}$. In this paper, we propose to find a function that associates the measure values of the property with the corresponding human assessments about it. As it was pointed out in section 5.1.1, thanks to the "homogeneity" in the presence degree of the property, each image $I_{i}^{p} \in \mathcal{I}^{p}$ can be seen as a set of sub-images with the same presence degree $v_{i}^{p}$ of the original one. From now on, we will note as $\mathcal{I}_{\mathcal{W}}^{p}=\left\{I_{i, w}^{p}, i=\right.$ $\left.1, \ldots, N_{p} ; w=1, \ldots, W\right\}$ the set of sub-images extracted from $\mathcal{I}^{p}$, where $I_{i, w}^{p}$ is the $w$-th sub-image of $I_{i}^{p}$ and $W$ is the number of sub-images considered for each image; on the other hand we will denote by $m_{p, k}^{i, w}$ the result of applying the measure $F_{k}^{p}$ to the sub-image $I_{i, w}^{p}$. According to this notation, let $\mathcal{I}_{\mathcal{W}}^{p, f i t} \subseteq \mathcal{I}_{\mathcal{W}}^{p}$ and $\mathcal{I}_{\mathcal{W}}^{p, \text { test }}=\mathcal{I}_{\mathcal{W}}^{p} \backslash \mathcal{I}_{\mathcal{W}}^{p, f i t}$ be two complementary subsets of $\mathcal{I}_{\mathcal{W}}^{p}$, that will be used for fitting the membership function and testing the obtained model, respectively.

Thus, in order to estimate the membership function that associates the measure values $\left(m_{p, k}^{i, w}\right)$ and the human assessments $\left(v_{i}^{p}\right)$, we propose to fit a suitable function to the subset of points:

$$
\Psi_{k}^{p, f i t}=\left\{\left(m_{p, k}^{i, w}, v_{i}^{p}\right) ; \forall I_{i, w}^{p} \in \mathcal{I}_{\mathcal{W}}^{p, f i t}\right\}
$$

In this paper, for each image $I_{i}^{p} \in \mathcal{I}^{p}, W=200$ sub-images of size $32 \times 32$ have been considered ${ }^{5}$, so $\mathcal{I}_{\mathcal{W}}^{p}$ is formed by 16000 sub-images. We propose to randomly select $75 \%$ of them for the fitting, so 12000 points are contained within $\Psi_{k}^{p, f i t}$.

The measure values can be affected by some factors, like brightness, contrast or noise, which typically causes outliers in the fitting points. For this reason, in our approach the membership function is calculated by means of a robust fitting of the multiset $\Psi_{k}^{p, f i t}$. In this modelling, the robust fitting based on Mestimators (a generalization of the least squares fitting) is used [37]. In addition, to define $\mathcal{T}_{k}^{p}$, the following considerations are taken into account:

- $\mathcal{T}_{k}^{p}$ should be a monotonic function.

- The values $\mathcal{T}_{k}^{p}(x)=0$ and $\mathcal{T}_{k}^{p}(x)=1$ should be reached.

Regarding the above properties, we propose to define $\mathcal{T}_{k}^{p}$ as a function of

\footnotetext{
${ }^{5}$ As mentioned in section 4, the measures used in this study are not size dependent. Therefore, the models obtained by means of the fitting process do not depend on the window size. Sub-images smaller than $32 \times 32$ are not considered because they would break texture primitives.
} 
Table 1: Fitting errors and test errors related to each measure for the properties of fineness (a), contrast (b) and directionality (c).

\begin{tabular}{|c|c|c|c|c|c|}
\hline $\begin{array}{l}\text { Fineness } \\
\text { measure }\end{array}$ & $\begin{array}{l}\text { Fitting } \\
\text { error }\end{array}$ & $\begin{array}{l}\text { Test } \\
\text { error }\end{array}$ & $\begin{array}{l}\text { Contrast } \\
\text { measure }\end{array}$ & $\begin{array}{l}\text { Fitting } \\
\text { error }\end{array}$ & $\begin{array}{l}\text { Test } \\
\text { error }\end{array}$ \\
\hline Amadasun & 0.1333 & 0.1695 & Tamura & 0.0340 & 0.0649 \\
\hline Correlation & 0.1401 & 0.1747 & Amadasun & 0.0780 & 0.1108 \\
\hline Abbadeni & 0.1639 & 0.1947 & Abbadeni & 0.1003 & 0.1393 \\
\hline FD & 0.1776 & 0.2084 & Haralick & 0.1157 & 0.1416 \\
\hline $\begin{array}{l}\text { Tamura } \\
\text { ED }\end{array}$ & $\begin{array}{l}0.1913 \\
0.2009\end{array}$ & $\begin{array}{l}0.2070 \\
0.2168\end{array}$ & \multicolumn{3}{|c|}{ (b) } \\
\hline DGD & 0.2031 & 0.2339 & & & \\
\hline $\mathrm{LH}$ & 0.2150 & 0.2336 & Directionality & Fitting & Test \\
\hline Weszka & 0.2157 & 0.2411 & measure & error & error \\
\hline Contrast & 0.2161 & 0.2475 & Tamura & 0.0687 & 0.1064 \\
\hline SNE & 0.2267 & 0.2418 & Abbadeni & 0.0788 & 0.1324 \\
\hline SRE & 0.2296 & 0.2455 & Fourier & 0.1073 & 0.1406 \\
\hline
\end{tabular}

(a)

(c)

the form ${ }^{6}$

$$
\mathcal{T}_{k}^{p}\left(x ; a_{n} \ldots a_{0}, \alpha, \beta\right)= \begin{cases}1 & x<\alpha \\ p o l y\left(x ; a_{n} \ldots a_{0}\right) & \alpha \leq x \leq \beta, \\ 0 & x>\beta\end{cases}
$$

with $\operatorname{poly}\left(x ; a_{n} \ldots a_{0}\right)$ being a polynomial function

$$
\operatorname{poly}\left(x ; a_{n} \ldots a_{0}\right)=a_{n} x^{n}+\ldots+a_{1} x^{1}+a_{0}
$$

In our proposal, the parameters $a_{n} \ldots a_{0}, \alpha$ and $\beta$ of the function $\mathcal{T}_{k}^{p}$ are calculated by carrying out a robust fitting on $\Psi_{k}^{p, f i t}$, with the constraint to obtain a strictly monotonic function between $\alpha$ and $\beta$. For the polynomial function, the cases of $n=1,2,3,4$ (i.e. linear, quadratic, cubic and quartic functions) have been considered.

The second column of tables 1(a), 1(b), and 1(c) show the least fitting error related to the measures used in this paper for the properties of fineness, contrast and directionality, respectively. Note that this value can be viewed as the goodness of each measure to represent the perception of the corresponding property. These tables have been ranked in increasing order of the fitting errors. In all the cases, the least error has been obtained for a polynomial function of order $n=3$ (the use of higher order functions does not provide better fits).

In addition, the test error for each measure has been calculated by using the subset of points $\Psi_{k}^{p, \text { test }}$ and it is shown in the third column of these tables. In

\footnotetext{
${ }^{6}$ Note that this function is defined for measures that decrease according to the presence of the property. For those that increase, the function needs to be changed appropriately, i.e. it takes the value 0 for $x<\beta$, it takes the value 1 for $x>\alpha$, and the polynomial function is computed for $\beta \leq x \leq \alpha$.
} 


\begin{tabular}{lccc}
\hline & $\begin{array}{c}\text { Fineness } \\
\text { (Amadasun) }\end{array}$ & $\begin{array}{c}\text { Contrast } \\
\text { (Tamura) }\end{array}$ & $\begin{array}{c}\text { Directionality } \\
\text { (Tamura) }\end{array}$ \\
\hline$a_{3}$ & -6.61279 & 1.6877 & -511.97 \\
$a_{2}$ & 9.49014 & -3.9536 & 1657.3 \\
$a_{1}$ & -6.48352 & 3.8763 & -1792.3 \\
$a_{0}$ & 1.87067 & -0.5728 & 648.14 \\
$\alpha$ & 0.17269 & 0.1775 & 0.8594 \\
$\beta$ & 0.58578 & 0.9620 & 0.9865 \\
\hline
\end{tabular}
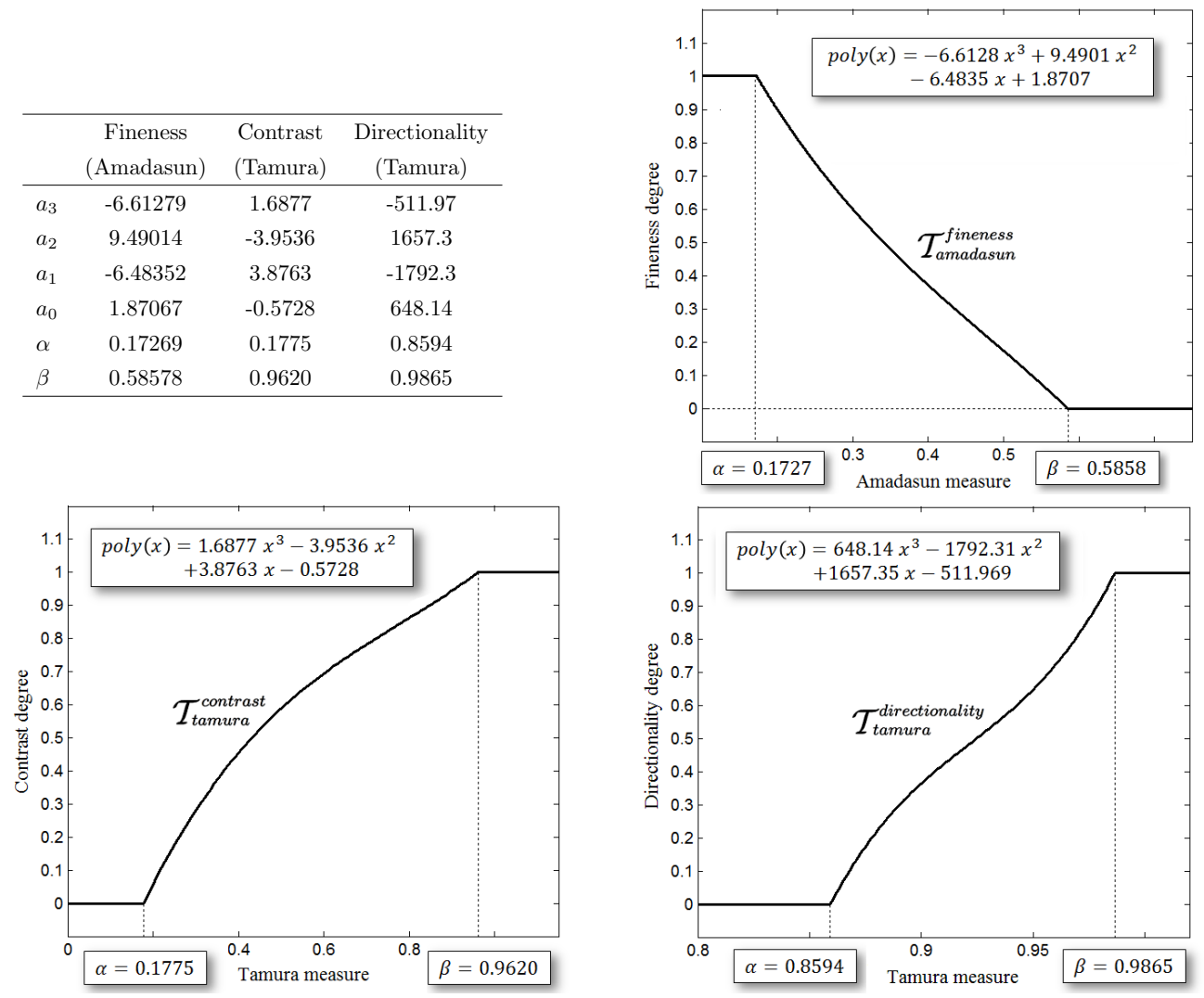

Figure 5: Proposed membership function $\mathcal{T}_{k}^{p}$ corresponding to fineness, contrast and directionality properties.

our approach, this error is calculated as the mean absolute difference between the values $v_{i}^{p}$ and the degrees obtained by applying the function $\mathcal{T}_{k}^{p}$ to the values $m_{p, k}^{i, w}$, for all the points $\left(m_{p, k}^{i, w}, v_{i}^{p}\right) \in \Psi_{k}^{p, t e s t}$, i.e.

$$
E_{k}^{p, \text { test }}=\frac{\sum_{\left(m_{p, k}^{i, w}, v_{i}^{p}\right) \in \Psi_{k}^{p, \text { test }}}\left|\mathcal{T}_{k}^{p}\left(m_{p, k}^{i, w}\right)-v_{i}^{p}\right|}{\operatorname{card}\left(\Psi_{k}^{p, t e s t}\right)}
$$

with $\operatorname{card}\left(\Psi_{k}^{p, \text { test }}\right)$ being the cardinality of $\Psi_{k}^{p \text {,test }}$.

In our experiments, the membership functions with the lowest error are obtained by using the measure of Amadasun in the case of fineness and the measures of Tamura in the case of contrast and directionality. Figure 5 shows the parameters and the graphical representation of these membership functions. 


\section{Adaptation to user's profiles and image con- text}

As it was pointed out, the non-adaptive fuzzy sets $\mathcal{T}_{k}^{p}$ obtained in section 5 are able to represent the presence degree of fineness, contrast and directionality in textures according to the average perception of these properties. These fuzzy sets can be considered as the default models for the fineness, contrast and directionality properties, because they can be used if additional information is not available (user's profile, context, etc.). However, a particular user may have a different perception of these properties, and, moreover, the image context may influence in the global perception, so an approach that allows to adapt the default models to a particular case is needed.

In this section, we propose a method to automatically adapt the membership function $\mathcal{T}_{k}^{p}$ to the different perception of the texture properties that a particular user can have (section 6.1) or the changes in perception influenced by the image context (section 6.2).

\subsection{Adaptation to user's profile}

In order to adapt the membership function to a particular user's perception about the property $p \in \mathcal{P}$, a set of texture images and its corresponding perception degree of this property should be provided by the user. Let $\mathcal{R}=$ $\left\{R^{1}, \ldots, R^{Z}\right\}$ be the set of $Z \geq 1$ texture images given by the user to represent his particular perception about the property, let $\mathcal{V}=\left\{v^{1}, \ldots, v^{Z}\right\}$ be the perception degrees associated to $\mathcal{R}$, and let $\mathcal{M}_{k}=\left\{m_{k}^{1}, \ldots, m_{k}^{Z}\right\}$ be the values for the measure $F_{k}^{p} \in \mathcal{F}^{p}$ applied to each image $R^{i} \in \mathcal{R}$. According to this notation, let $\Omega_{k}=\left\{\left(m_{k}^{i}, v^{i}\right), m_{k}^{i} \in \mathcal{M} ; v^{i} \in \mathcal{V} ; m_{k}^{i}<m_{k}^{i+1}\right\}_{i=1, \ldots, Z}$ be the set of pairs that associate each measure value with its corresponding perception degree (called as adaptation points in the following) ordered by the measure value.

We propose to obtain $\hat{\mathcal{T}}_{k}^{p}$ by means of a transformation that adapts the membership function $\mathcal{T}_{k}^{p}$ to the new criteria $\Omega_{k}$. This transformation is performed by translating and expanding (or compressing) $\mathcal{T}_{k}^{p}$ in the domain of the measure in order to force $\hat{\mathcal{T}}_{k}^{p}$ to go through the adaptation points. Notice that the proposed adaptation method keeps the tendency of the non-adaptive membership function, i.e. the function increases and decreases according to the average human perception. Thus, we propose to define $\hat{\mathcal{T}}_{k}^{p}$ as a function of the form $^{7}$

\footnotetext{
${ }^{7}$ Notice that the transformation for $x \leq m_{k}^{1}$ and $x \leq m_{k}^{2}$ is the same. In fact, as $m_{k}^{1}>m_{k}^{2}$, the first condition can be reduced to $x \leq m_{k}^{2}$. The same happens with $x>m_{k}^{Z-1}$ and $x>m_{k}^{Z}$, that can be reduced to $x>m_{k}^{Z-1}$.
} 


$$
\hat{\mathcal{T}}_{k}^{p}\left(x ; \Omega_{k}\right)= \begin{cases}\mathcal{T}_{k}^{p}\left(\mathbf{A}_{m_{k}^{1} m_{k}^{2}}^{\bar{m}_{1}^{1} \bar{m}_{k}^{2}}(x)\right) & x \leq m_{k}^{1} \text { or } x \leq m_{k}^{2}, \\ \vdots & \\ \mathcal{T}_{k}^{p}\left(\mathbf{A}_{m_{k}^{i} m_{k}^{i+1}}^{\bar{m}_{k}^{i} \bar{m}_{k}^{i+1}}(x)\right) & m_{k}^{i}<x \leq m_{k}^{i+1}, \\ \vdots & \\ \mathcal{T}_{k}^{p}\left(\mathbf{A}_{m_{k}^{Z} m_{k}^{Z-1} m_{k}^{Z}}^{\bar{m}^{Z}-1} \bar{m}_{Z}^{Z}\right. & \\ & x>m_{k}^{Z-1} \text { or } x>m_{k}^{Z}\end{cases}
$$

with $\mathcal{T}_{k}^{p}$ being the function defined in Eq. (6), with $\bar{m}_{k}^{i}=\left(\mathcal{T}_{k}^{p}\right)^{-1}\left(v^{i}\right) \forall i$, and where $\mathbf{A}_{a^{\prime} b^{\prime}}^{a b}(x)$ is defined as a translation and expansion function of the form

$$
\mathbf{A}_{a^{\prime} b^{\prime}}^{a b}(x)=\frac{x-a^{\prime}}{b^{\prime}-a^{\prime}}(b-a)+a
$$

It should be noticed that, since $\mathcal{T}_{k}^{p}$ is a bijective function on $[\alpha, \beta]$ (it is surjective and strictly monotone on $[\alpha, \beta]$ ), the inverse function $\left(\mathcal{T}_{k}^{p}\right)^{-1}$ always exists. In our implementation, the Newton-Raphson method has been applied in order to obtain the values $\bar{m}_{k}^{i}$.

By considering the definition of $\mathcal{T}_{k}^{p}$ given by Eq. (6), the adapted membership function $\hat{\mathcal{T}}_{k}^{p}$ defined in Eq. (9) can be represented as:

$$
\hat{\mathcal{T}}_{k}^{p}\left(x ; \Omega_{k}\right)= \begin{cases}1 & x<\hat{\alpha}, \\ \operatorname{poly}\left(\mathbf{A}_{m_{k}^{1} m_{k}^{2}}^{\bar{m}_{k}^{1} \bar{m}_{k}^{2}}(x)\right) & \hat{\alpha} \leq x \leq m_{k}^{2}, \\ \vdots & \\ \operatorname{poly}\left(\mathbf{A}_{m_{k}^{i} m_{k}^{i+1}}^{\bar{m}_{i}^{i} \bar{m}^{i+1}}(x)\right) & m_{k}^{i}<x \leq m_{k}^{i+1}, \\ \vdots & \\ \operatorname{poly}\left(\mathbf{A}_{m_{k}^{Z}}^{\bar{m}_{k}^{Z-1} m_{k}^{Z}} \bar{m}_{k}^{Z}(x)\right) & m_{k}^{Z-1}<x \leq \hat{\beta}, \\ 0 & x>\hat{\beta}\end{cases}
$$

where $\hat{\alpha}$ and $\hat{\beta}$ are calculated (if they are not defined in $\Omega_{k}$ ) $\operatorname{as}^{8}$

$$
\begin{gathered}
\hat{\alpha}=\mathbf{A}_{\bar{m}_{k}^{1} \bar{m}_{k}^{2}}^{m_{1}^{1} m^{2}}(\alpha) \\
\hat{\beta}=\mathbf{A}_{\bar{m}_{k}^{Z-1} \bar{m}_{k}^{Z}}^{m_{Z-1}^{Z-1} m^{Z}}(\beta)
\end{gathered}
$$

with $\alpha, \beta$ and poly being the values and the polynomial function defined in Eq. (6). Note that poly is the same polynomial function in all the parts of Eq. (11).

It should be noticed that Eq. (9) and Eq. (11) are valid only for $Z>1$. In the particular case of $Z=1$ only a translation is performed

\footnotetext{
${ }^{8}$ For measures that decrease according to the perception of the property. For those that increases, $\hat{\alpha}$ is calculated by applying $\alpha$ to Eq. (13) and $\hat{\beta}$ is calculated by applying $\beta$ to Eq. (12).
} 


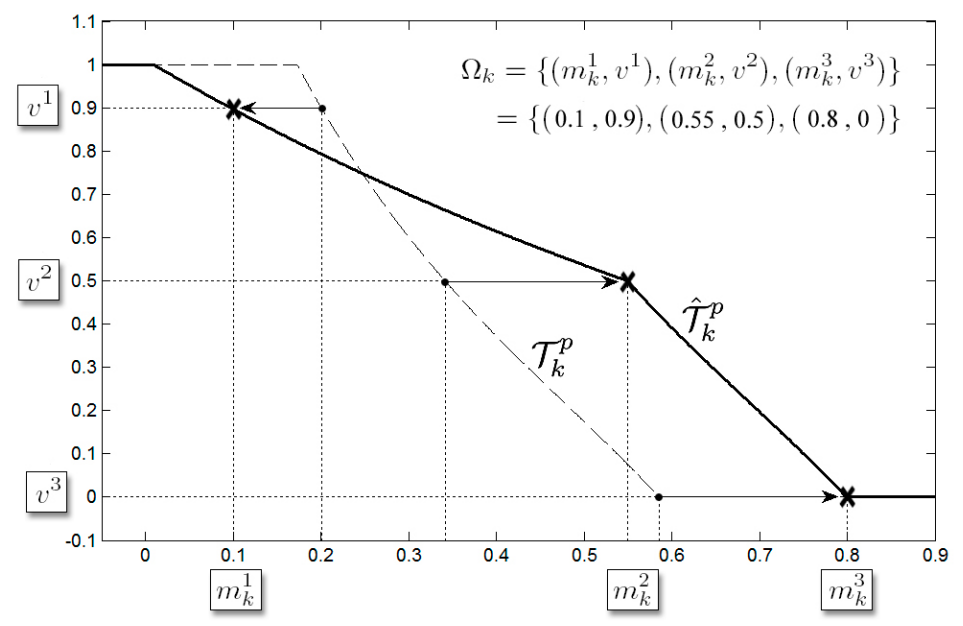

Figure 6: Example of adaptation to a new user's perception. The adapted model $\hat{\mathcal{T}}_{k}^{p}$ (solid line) is obtained by adapting the non-adaptive model $\mathcal{T}_{k}^{p}$ (dashed line) according to $\Omega_{k}=\left\{\left(m_{k}^{i}, v^{i}\right)\right\}_{i=1, \ldots, Z}$ with $Z=3$.

$$
\hat{\mathcal{T}}_{k}^{p}\left(x ; \Omega_{k}\right)=\mathcal{T}_{k}^{p}\left(x+\bar{m}_{k}^{1}-m_{k}^{1}\right)
$$

It should be noticed that the model obtained with the proposed adaptive method is not the same as if the we calculate the non-adaptive model using the opinion of the new user in conjunction with the assessments given by the original users. The non-adaptive model reflects the opinion of the majority of the subjects, so it would only reflect the perception of the new user if this perception matched with the opinion of the majority.

An example of the adaptation method presented in this section is shown in Figure 6. The proposed transformation is applied to the non-adaptive model $\mathcal{T}_{k}^{p}$ for the fineness property (dashed line) in order to obtain the adapted model $\hat{\mathcal{T}}_{k}^{p}$ (solid line), according to $\Omega_{k}$. The non-adaptive model for the fineness measure of Amadasun, that is defined by the parameter values shown in Figure 5, has been used in this example:

$$
\mathcal{T}_{k}^{p}(x)= \begin{cases}1 & x<0.17269 \\ \operatorname{poly}(x) & 0.17269 \leq x \leq 0.58578 \\ 0 & x>0.58578\end{cases}
$$

with $\operatorname{poly}(x)=-6.6128 x^{3}+9.4901 x^{2}-6.4835 x+1.8707$.

We suppose that a new user gives three texture images to represent his particular fineness perception. Let's assume that the values of the fineness measure $F_{k}^{p}$ for the three images are $m_{k}^{1}=0.1, m_{k}^{2}=0.55$ and $m_{k}^{3}=0.8$. Let $v^{1}=0.9, v^{2}=0.5$ and $v^{3}=0$ be the perception degree of fineness given by the 
user to these images. The first point $(0.1,0.9)$ imposes that the value $v^{1}=0.9$, that is achieved by $\mathcal{T}_{k}^{p}$ in $\bar{m}_{k}^{1}=0.201$, should be achieved by $\hat{\mathcal{T}}_{k}^{p}$ in $m_{k}^{1}=0.1$. In the same way, the values $v^{2}=0.5$ and $v^{3}=0$, that are achieved by the nonadaptive model in $\bar{m}_{k}^{2}=0.342$ and $\bar{m}_{k}^{3}=0.586$ respectively, should be achieved by the adapted model in $m_{k}^{2}=0.55$ and $m_{k}^{3}=0.8$. Thus, the adapted model $\hat{\mathcal{T}}_{k}^{p}$ is obtained using Eq. (11) as:

$$
\begin{aligned}
& \hat{\mathcal{T}}_{k}^{p}\left(x ; \Omega_{k}\right)= \begin{cases}1 & x<0.027 \\
\operatorname{poly}\left(\mathbf{A}_{0.10 .55}^{0.2010 .342}(x)\right) & 0.027 \leq x \leq 0.55, \\
\operatorname{poly}\left(\mathbf{A}_{0.550 .8}^{0.3420 .586}(x)\right) & 0.55<x \leq 0.8 \\
0 & x>0.8\end{cases} \\
&= \begin{cases}1 & x<0.027 \\
\operatorname{poly}\left(\frac{0.141 x}{0.45}+0.170\right) & 0.027 \leq x \leq 0.55 \\
\operatorname{poly}\left(\frac{0.244 x}{0.25}-0.195\right) & 0.55 \geq x>0.8 \\
0 & x>0.8\end{cases}
\end{aligned}
$$

In this case, the value $\hat{\beta}$ has been defined by the user in $\Omega_{k}(\hat{\beta}=0.8)$, and the value $\hat{\alpha}$ is calculated as

$$
\hat{\alpha}=\mathbf{A}_{0.10 .55}^{0.2010 .342}(0.1727)=0.027
$$

\subsection{Adaptation to image context}

Natural images will usually show several textures with different perception degrees of the properties. It is natural to assume that the textures with the minimum and the maximum presence of a property in the image may influence the perception of this property for the rest of textures, i.e. the perception can depend on the context. For example, in the case of the fineness property, the coarsest and the finest texture in the image may inhibit the rest of textures, influencing their perception of fineness.

In this section, a proposal for adapting the membership function $\mathcal{T}_{k}^{p}$ to the image context is presented. In our approach, the minimum and the maximum perception degrees of the property $p \in \mathcal{P}$, i.e. the values where the function $\hat{\mathcal{T}}_{k}^{p}$ achieves the membership degrees 0 and 1 , noted as $\hat{\beta}$ and $\hat{\alpha}$, will depend on the inhibition present in the image. In turn, this inhibition will depend on the difference between the textures with the minimum and the maximum presence of the property in the image, in the sense that the greater this difference, the stronger the inhibition. From now on, we will denote by $\mathbf{M}_{k}^{\text {min }}$ and $\mathbf{M}_{k}^{\max }$ the value of the measure $F_{k}^{p}$ for the textures with the minimum and the maximum presence of the property in the image, and we will denote by $\lambda_{k}^{p}$ the inhibition degree present in the image for the property $p \in \mathcal{P}$ according to the measure $F_{k}^{p}$. At this point, our first aim is to obtain the values $\mathbf{M}_{k}^{\text {min }}$ and $\mathbf{M}_{k}^{\text {max }}$ of the corresponding image. Secondly, these values will be used to estimate the inhibition degree $\lambda_{k}^{p}$. Finally, this inhibition degree will be used to calculate the values $\hat{\beta}$ and $\hat{\alpha}$ that will impose the adaptation to the image context. 
In order to obtain the textures with the minimum and the maximum presence of the property in the image, for each pixel in the original image, the value of the measure $F_{k}^{p}$ is calculated using a centered window of size $32 \times 32$. Let $\mathcal{M}=\left\{m_{k}^{i}, m_{k}^{i} \leq m_{k}^{i+1}\right\}_{i=1, \ldots, N}$ be the ordered set of these values. The textures with the minimum and the maximum presence of the property will correspond with the first and the last element in this set ${ }^{9}$, respectively, i.e. $\mathbf{M}_{k}^{\min }=m_{k}^{1}$ and $\mathbf{M}_{k}^{\max }=m_{k}^{N}$. However, in order to avoid the influence of outliers (the presence of very low and very large measure values), the elements $z>1$ and $z^{\prime}<N$ have been chosen, i.e. $\mathbf{M}_{k}^{\min }=m_{k}^{z}$ and $\mathbf{M}_{k}^{\max }=m_{k}^{z^{\prime}}$. In particular, we propose to use the $20^{t h}$ percentile and the $80^{t h}$ percentile in $\mathcal{M}$, i.e. $z=\operatorname{round}(0.2 N+0.5)$ and $z^{\prime}=\operatorname{round}(0.8 N+0.5)$, with $\operatorname{round}(x)$ being the function that returns the nearest integer to $x$.

Once the values $\mathbf{M}_{k}^{\min }$ and $\mathbf{M}_{k}^{\max }$ are calculated, the next step is to estimate the inhibition degree $\lambda_{k}^{p}$ present in the image. In this paper, we consider that $\lambda_{k}^{p}$ will reach the highest degree $\left(\lambda_{k}^{p}=1\right)$ if the difference $\left|\mathbf{M}_{k}^{\max }-\mathbf{M}_{k}^{\text {min }}\right|$ is large enough. Thus, we propose to define the inhibition degree as a value between 0 and 1 of the form

$$
\lambda_{k}^{p}= \begin{cases}\frac{\left|\mathbf{M}_{k}^{\max }-\mathbf{M}_{k}^{\text {min }}\right|}{U_{k}} & \left|\mathbf{M}_{k}^{\max }-\mathbf{M}_{k}^{\min }\right|<U_{k}, \\ 1 & \left|\mathbf{M}_{k}^{\text {max }}-\mathbf{M}_{k}^{\text {min }}\right| \geq U_{k}\end{cases}
$$

with $U_{k}$ being the threshold value for considering that the difference between the textures with the maximum and the minimum presence of the property in the image is large enough. In our approach, we consider that the difference between textures with membership degrees 0 and 1 according to the non-adaptive model is large enough. Thus, we propose to define this threshold as

$$
U_{k}=|\alpha-\beta|
$$

with $\alpha$ and $\beta$ being the values defined in Eq. (6).

At this point, the aim is to obtain $\hat{\alpha}$ and $\hat{\beta}$ on the basis of the inhibition degree. In our approach, if the inhibition is strong $\left(\lambda_{k}^{p}=1\right), \hat{\alpha}$ and $\hat{\beta}$ will be imposed by the textures with the maximum and the minimum presence of the property in the image, i.e. $\hat{\alpha}=\mathbf{M}_{k}^{\max }$ and $\hat{\beta}=\mathbf{M}_{k}^{\text {min }}$. If no inhibition is present in the image $\left(\lambda_{k}=0\right), \hat{\alpha}$ and $\hat{\beta}$ will coincide with the corresponding values of the non-adaptive model, i.e. $\hat{\alpha}=\alpha$ and $\hat{\beta}=\beta$. Thus, in general, we propose to calculate $\hat{\alpha}$ and $\hat{\beta}$ as

$$
\begin{aligned}
& \hat{\alpha}=\alpha+\lambda_{k} \cdot\left(\mathbf{M}_{k}^{\max }-\alpha\right) \\
& \hat{\beta}=\beta+\lambda_{k} \cdot\left(\mathbf{M}_{k}^{\min }-\beta\right)
\end{aligned}
$$

This way, the membership function $\hat{\mathcal{T}}_{k}^{p}$ adapted to the image context can be obtained by applying the same transformation shown in section 6.1 on the basis of the set $\Omega_{k}=\{(\hat{\alpha}, 1),(\hat{\beta}, 0)\}$.

\footnotetext{
${ }^{9}$ For measures that increase according to the perception of the property.
} 


\section{$7 \quad$ Results}

In this section, the goodness of the fuzzy models proposed for fineness, contrast and directionality properties will be analyzed. First, in section 7.1, we will compare them with the state of the art in the modelling of visual texture properties. Then, the proposed fuzzy sets will be applied to several examples with images in order to analyze its performance. In section 7.2, the non-adaptive models will be directly applied, while in sections 7.3 and 7.4 they will be previously adapted to new user's perceptions and the image context, respectively. In particular, the fuzzy sets with least fitting error and least test error (shown in Figure 5) will be used.

\subsection{Comparison with the state of the art}

At this point, the aim is to compare the non-adaptive fuzzy sets obtained in section 5 with the state of the art in the modelling of visual texture properties. As has been commented throughout this paper, the proposed fuzzy models allow us to represent the degree to which a texture property is present, and we have verified by means of goodness measures (fitting errors and test errors) that the obtained degrees match with the human perception of the property. However, from our knowledge, there is no other fuzzy approach in the literature with the ability of providing this type of information. Nevertheless, as has been shown in section 1, most of the existing measures allow to represent a textural property in the sense that the greater the value given by the measure, the greater (lower) the presence of this property. With this in mind, and in order to compare the different approaches, we will study whether the increase in the value of a measure is always produced by the increase (or decrease) in the presence of the corresponding property.

In particular, two comparative studies will be performed using the subset of images $\mathcal{I}_{\mathcal{W}}^{p, t e s t}$. In the first one, we will study the correlation between the order given by the measure values computed from the images in $\mathcal{I}_{\mathcal{W}}^{p, t e s t}$ and the order of these images according to the poll ${ }^{10}$. To do this, we propose to use the Kendall rank correlation coefficient [38] and the Spearman rank correlation coefficient [39], that are widely used in this type of problems. The Kendall coefficient is defined as:

$$
\tau=\frac{n_{c}-n_{d}}{n(n-1) / 2}
$$

with $n$ being the number of ranked elements, and where $n_{c}$ and $n_{d}$ are the number of concordant and discordant pairs comparing both rankings, as it is shown in [38]. The Spearman coefficient is defined as:

$$
\rho=\frac{\sum_{i}\left(x_{i}-\bar{x}\right)\left(y_{i}-\bar{y}\right)}{\sqrt{\sum_{i}\left(x_{i}-\bar{x}\right)^{2} \sum_{i}\left(y_{i}-\bar{y}\right)^{2}}}
$$

\footnotetext{
${ }^{10}$ Note that in this case we are not interested in the specific assessment associated to each image, but in the ranking of these values.
} 
Table 2: Comparative study of rank correlation by means of Spearman and Kendall coefficients.

\begin{tabular}{|c|c|c|c|c|c|}
\hline $\begin{array}{l}\text { Fineness } \\
\text { model }\end{array}$ & $\begin{array}{l}\text { Spearman } \\
\text { coefficient }\end{array}$ & $\begin{array}{c}\text { Kendall } \\
\text { coefficient }\end{array}$ & $\begin{array}{l}\text { Contrast } \\
\text { model }\end{array}$ & $\begin{array}{l}\text { Spearman } \\
\text { coefficient }\end{array}$ & $\begin{array}{l}\text { Kendall } \\
\text { coefficient }\end{array}$ \\
\hline Chamorro & 0.7303 & 0.5705 & Chamorro & 0.9548 & 0.8633 \\
\hline Amadasun & 07299 & 05660 & Tamura & 0.9504 & 0.8415 \\
\hline Amadasun & 0.7299 & 0.5660 & FuzzyP & 0.9317 & 0.8592 \\
\hline Correlation & 0.7289 & 0.5610 & Amadasun & 0.8777 & 0.7358 \\
\hline Abbadeni & 0.6981 & 0.5293 & Abbadeni & 0.8365 & 0.6888 \\
\hline $\mathrm{ED}$ & 0.6407 & 0.4744 & Haralick & 0.8262 & 0.6744 \\
\hline FD & 0.6163 & 0.4545 & \multirow{2}{*}{\multicolumn{3}{|c|}{ (b) }} \\
\hline Tamura & 0.6118 & 0.4567 & & & \\
\hline FuzzyP & 0.5983 & 0.4781 & \multirow{2}{*}{$\begin{array}{l}\text { Directionality } \\
\text { model }\end{array}$} & \multirow{2}{*}{$\begin{array}{l}\text { Spearman } \\
\text { coefficient }\end{array}$} & \multirow{2}{*}{$\begin{array}{c}\text { Kendall } \\
\text { coefficient }\end{array}$} \\
\hline DGD & 0.5629 & 0.4197 & & & \\
\hline $\mathrm{LH}$ & 0.5624 & 0.4132 & Chamorro & 0.8986 & 0.7747 \\
\hline Weszka & 0.5436 & 0.4039 & Tamura & 0.8961 & 0.7582 \\
\hline SRE & 0.5413 & 0.3984 & FuzzyP & 0.8696 & 0.7716 \\
\hline Contrast & 0.4923 & 0.3674 & Abbadeni & 0.8387 & 0.6913 \\
\hline SNE & 0.4857 & 0.3555 & Fourier & 0.8063 & 0.6531 \\
\hline
\end{tabular}

(a)

(c)

with $x_{i}$ and $y_{i}$ being the rank of each pair of elements in its corresponding ranking, and with $\bar{x}$ and $\bar{y}$ being the mean of the values in each ranking. In both cases, a higher coefficient indicates a better correlation between the ranking of the measure values and the ranking of the human assessments about the presence of the property.

In the second comparative study, the significance of each model will be analyzed. In this case, we study whether the values given by the model for textures with different presence of the property are significantly different compared to the values obtained from textures with a similar presence. For this purpose, the F-statistic [40] will be computed from the $R^{p}$ classes in which the images in $\mathcal{I}_{\mathcal{W}}^{p, t e s t}$ have been grouped in the poll. This statistic compares the between-group variation of the values with their within-group variation as follows:

$$
F=\frac{\sum_{i=1}^{k} n_{i}\left(\bar{x}_{i}-\bar{x}\right)^{2} /(k-1)}{\sum_{i=1}^{k} \sum_{j=1}^{n}\left(x_{i j}-\bar{x}_{i}\right)^{2} /(N-k)}
$$

where $k$ is the total number of groups, $n_{i}$ is the number of elements in the $i$-th group, $N$ is the total numer of elements, $x_{i j}$ represents the $j$-th element in group $i, \bar{x}_{i}$ is the mean in group $i$, and $\bar{x}$ is the mean of all the elements. A higher F-statistic means that the model has more significance, i.e. it allows to distinguish more clearly different degrees to which the property is present.

In these two studies, we will compare the non-adaptive fuzzy models proposed in this paper (the models with the highest goodness according to fitting and test errors) with the state of the art in the modelling of texture properties, 
Table 3: Comparative study of significance by means of the F-statistic.

\begin{tabular}{|c|c|c|c|}
\hline $\begin{array}{l}\text { Fineness } \\
\text { model }\end{array}$ & $\begin{array}{l}\text { Significance } \\
\text { (F-statistic) }\end{array}$ & $\begin{array}{l}\text { Contrast } \\
\text { model }\end{array}$ & $\begin{array}{l}\text { Significance } \\
\text { (F-statistic) }\end{array}$ \\
\hline Chamorro & 168.7424 & Chamorro & 2406.6 \\
\hline Correlation & 155.9657 & FuzzyP & 1556.2 \\
\hline Tamura & 125.0183 & Tamura & 1268.8 \\
\hline Amadasun & 118.1959 & Amadasun & 427.36 \\
\hline FuzzyP & 115.7747 & Haralick & 248.86 \\
\hline FD & 114.4741 & Abbadeni & 211.06 \\
\hline $\mathrm{ED}$ & 110.3823 & \multicolumn{2}{|c|}{ (b) } \\
\hline DGD & 70.7770 & & \\
\hline $\mathrm{LH}$ & 69.6480 & model & (F-statistic) \\
\hline Weszka & 65.5035 & Chamorro & 592.51 \\
\hline SNE & 66.7699 & Tamura & 589.60 \\
\hline $\mathrm{SRE}$ & 53.0102 & FuzzyP & 477.98 \\
\hline Abbadeni & 42.4382 & Abbadeni & 325.89 \\
\hline Contrast & 34.0349 & Fourier & 247.68 \\
\hline
\end{tabular}

(a)

(c)

consisting of all the crisp measures shown in section 4, as well as the fuzzy approaches commented in section 2. Note that these fuzzy approaches are based on partitions, so they do not provide a presence degree of the texture property, but a set of linguistic labels (and their corresponding membership degrees) to describe this property. Thus, this information cannot be used directly in the proposed comparative studies. However, from a semantical point of view, this information also provides a presence estimation of the texture properties, although it is represented in a different mathematical format. In this sense, it can be ranked on the basis of the linguistic labels, that are semantically ordered according to the presence of the property. This way, given an image from the set $\mathcal{I}_{\mathcal{W}}^{p \text {,test }}$, we associate it to the linguistic label with the highest membership degree, obtaining a ranking that can be used in the comparative studies. As has been commented in section 2, the majority of the fuzzy approaches in the literature $[10,22,4,41,23]$ propose, for each texture property, a fuzzy partition with five linguistic terms defined on the domain of the corresponding Tamura measure. According to these approaches, we have generated each fuzzy partition through an unsupervised fuzzy clustering algorithm on the basis of the measure values obtained from the subset of images $\mathcal{I}_{\mathcal{W}}^{p, f i t}$. As all these approaches propose a similar solution, we have included the item "FuzzyP" in the comparative studies, that represents all them.

Tables 2 and 3 show the results obtained in these two studies for the properties of coarseness, contrast and directionality. The first table is ranked in decreasing order of the Spearman rank correlation coefficient, while the second one is ranked in decreasing order of the F-statistic. As can be seen, the best 
(a)
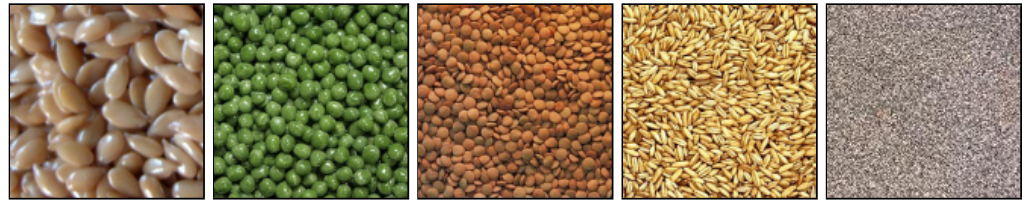

(b)
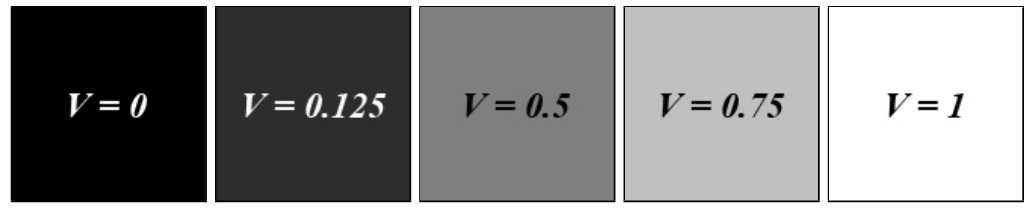

(c)
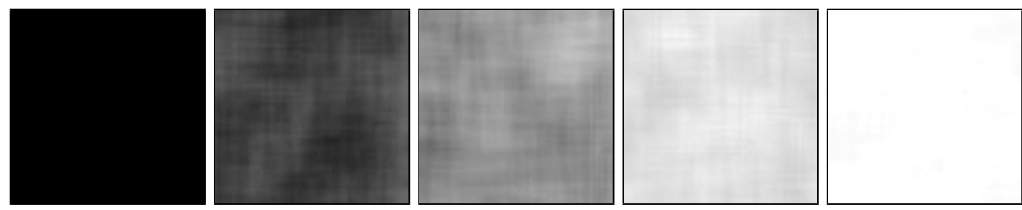

Figure 7: Experiment using the non-adaptive fuzzy model for the fineness property. (a) Collection of texture images. (b) Human assessments about the presence degree of fineness. (c) Membership degrees obtained by applying the proposed fineness model.

approach in all the comparative studies is the non-adaptive fuzzy model proposed in this paper. It may be noticeable that the values of both correlation coefficients for the fineness property (Table 2(a)) are relatively low. This is due only to the fact that nine different classes of fineness have been considered in the poll and there is much overlap between them, which is also reflected in the corresponding study of significance (Table 3(a)).

Therefore, although the full potential of our approach is not being used in these comparative studies, the obtained results show that our approach already improves the state of the art. In order to appreciate the ability of our fuzzy models to represent the presence degree of a texture property, which is not reflected in these comparative studies, the results obtained by applying these models to experiments with different images will be shown in next sections.

\section{$7.2 \quad$ Non-adaptive modelling}

In this section, several experiments using the proposed non-adaptive fuzzy set has been directly applied, without taking into account the particular perception of a new user or the image context. For the first one, we have considered Figure $7(\mathrm{a})$, corresponding to a collection of texture images, each one with a different increasing perception degree of fineness. These images are part of the set used in the poll, so human assessments about fineness presence are available in order to compare with the obtained results.

Figure $7(\mathrm{~b})$ shows an ideal mapping from the original texture images to 
(a)
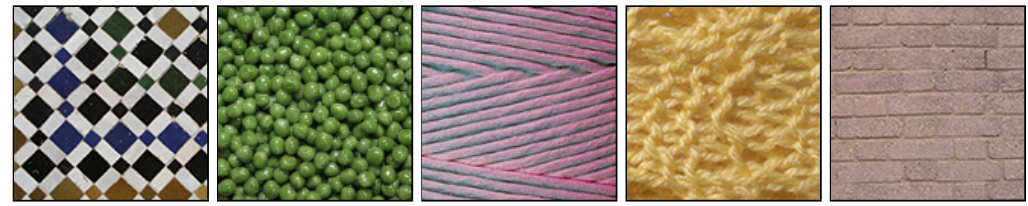

(b)
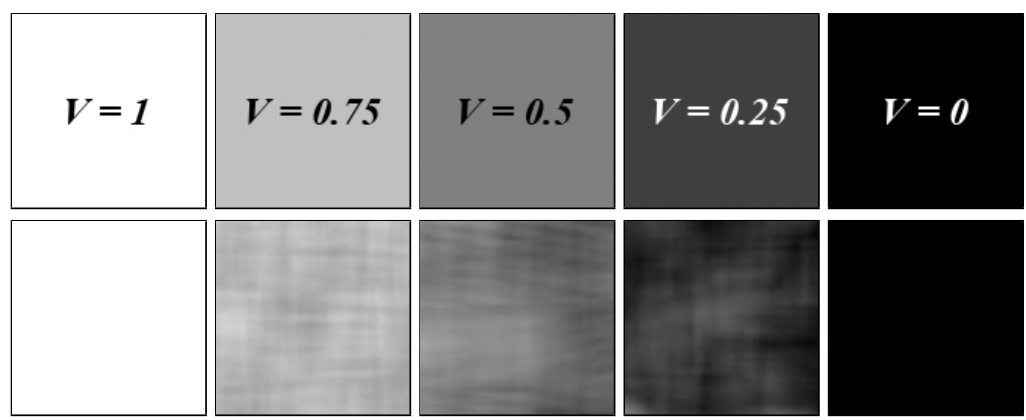

Figure 8: Experiment using the non-adaptive fuzzy model for the contrast property. (a) Collection of texture images. (b) Human assessments about the presence degree of contrast. (c) Membership degrees obtained by applying the proposed contrast model.

their fineness values, where all pixels corresponding to the same texture image have been mapped using the human assessment associated to this image. These assessments (between 0 and 1) have been mapped into a gray level from 0 to 255 , so that a white pixel in the mapping indicates maximum perception of fineness, while a black one indicates no perception of fineness (maximum perception of coarseness).

Figure 7(c) shows a mapping from the original texture images to their fineness values obtained by applying the proposed fineness model. For each pixel in the original images, a centered window of size $32 \times 32$ has been analyzed and its fineness membership degree has been calculated using the corresponding model. This degree has been mapped into a gray level from 0 to 255. It can be noticed that our model captures the evolution of the perception degrees of fineness, and the obtained mappings, that represents the estimated presence degree of this property, can be directly interpreted by humans.

Two similar experiments, shown in figures 8 and 9 , have been performed for the properties of contrast and directionality, respectively. In the case of contrast, we have considered the collection of texture images shown in Figure 8(a), each one with a different decreasing perception degree of contrast. As in the previous experiment, these images are part of the set used in the corresponding poll, so human assessments about contrast presence are available in order to compare with the obtained results.

Figure 8(b) shows an ideal mapping from the original texture images to their contrast values, where all pixels corresponding to the same texture image have 
(a)
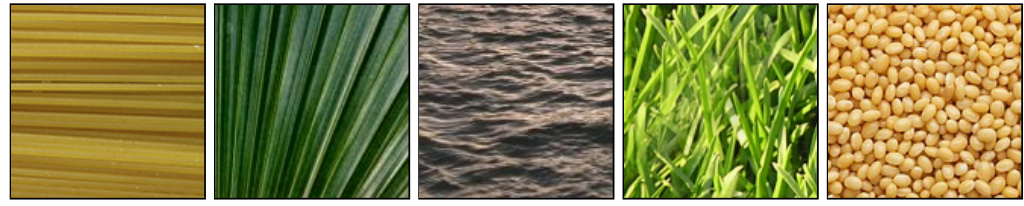

(b)
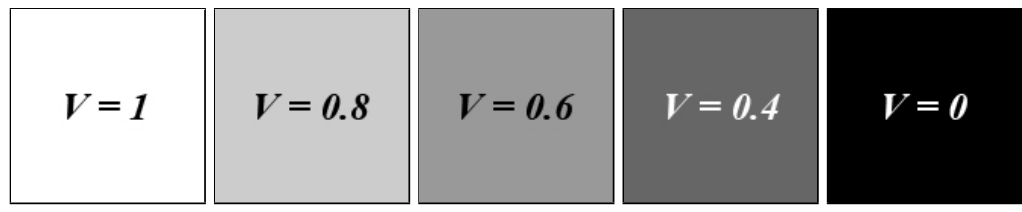

(c)
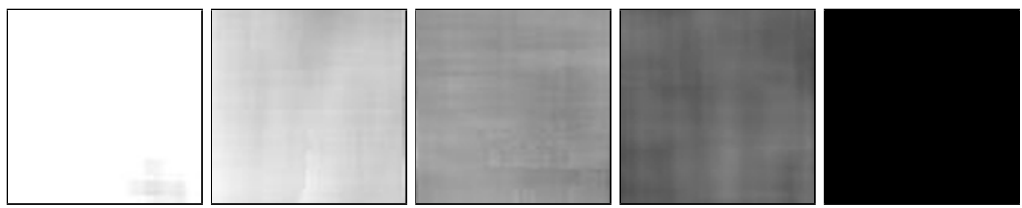

Figure 9: Experiment using the non-adaptive fuzzy model for the directionality property. (a) Collection of texture images. (b) Human assessments about the presence degree of directionality. (c) Membership degrees obtained by applying the directionality model.

been mapped using the human assessment associated to this image. In this case, a white pixel in the mapping indicates maximum perception of contrast, while a black one indicates no perception of this property.

Figure 8(c) shows a mapping from the original texture images to their contrast values obtained by applying the proposed contrast model, which is calculated in a similar way to the fineness one. It can be noticed that the result obtained with our model matches what a human would expect, capturing the evolution of the perception degrees of contrast.

In the case of directionality, we have considered the collection of texture images shown in Figure 9(a), each one with a different decreasing perception degree of directionality. As in the previous experiments, these images are part of the set used in the corresponding poll, so human assessments about directionality presence are available in order to compare with the results.

Figure 9(b) shows an ideal mapping from the original texture images to their directionality values, where all pixels corresponding to the same texture image have been mapped using the human assessment associated to this image. In this case, a white pixel in the mapping indicates maximum perception of directionality, while a black one indicates no perception of this property (omnidirectional texture).

Figure 9(c) shows a mapping from the original images to their directionality values obtained by applying the proposed directionality model, which is calculated in a similar way to the previous ones. By comparing with the ideal 
mapping of Figure 9(b), it can be noticed that the proposed model captures the evolution of the perception degrees of directionality, keeping a close resemblance to what a human would expect.

In the next three experiments, the proposed non-adaptive fuzzy set has been employed for pattern recognition. Figures 10(a), 10(c) and 10(e) show the images that have been used for the experiments corresponding to the properties of fineness, contrast and directionality, respectively. The first one is a microscopic image of a premature infant's lung tissue affected by the hyaline membrane disease. We want to separate the collapsed alveoli presents in the image, that corresponds to the uniform light region, from the rest of lung tissue. The brightness values in regions of the original image are not distinct, so texture information is needed for extracting the uniform areas. Figure 10(b) shows a mapping from the original image to its fineness membership degrees using the proposed model. It can be noticed that uniform regions correspond to areas with low degrees of fineness (i.e., high coarseness), so if only the pixels with fineness degree lower than 0.1 are selected (which is equivalent to a coarseness degree upper than 0.9 , i.e. an $\alpha$-cut of the coarseness fuzzy set with $\alpha=0.9$ ), the collapsed alveoli emerge with ease.

Figure 10(c) shows an image of two tires with different wear levels. The tire on the right has deep grooves, while the one on the left has an irregular wear in the center and on one side. The contrast of a visual texture is related to the depth perception and the relief of the corresponding physical texture: textures with high relief in nature may appear with high contrast in the image (due to the illumination effect). Thus, similar physical textures with different relief, can be distinguished in an image by analyzing the contrast of the corresponding visual textures. Figure 10(d) shows a mapping from the original image to its contrast values using the proposed model. It can be noticed that the worn parts correspond to areas with low contrast degrees, so they can be identified if only the pixels with contrast degree lower than 0.1 are selected.

Figure 10(e) shows an image of a piece of wood with a knot in it. Imperfections like knots could affect the technical properties of wood, as well as its visual aspect, so it is necessary to identify their presence. The wooden panels employed in construction and furniture manufacturing usually have a texture with high directionality, due to the wood grain, but the directionality associated to the knot area is very low. Figure 10(f) shows a mapping from the original image to its directionality values using the proposed model. If only the pixels with directionality degree lower than 0.1 are selected, the knot can be automatically identified.

\subsection{Adaptation to users' perception}

In this section, several experiments using the proposed fuzzy sets adapted to the particular perception of different users are shown. For the first one, we have considered Figure 11(a), corresponding to a natural image where several textures with different perception degrees of fineness are shown. Figure 11(d) shows a mapping from the original image to fineness values using the proposed 


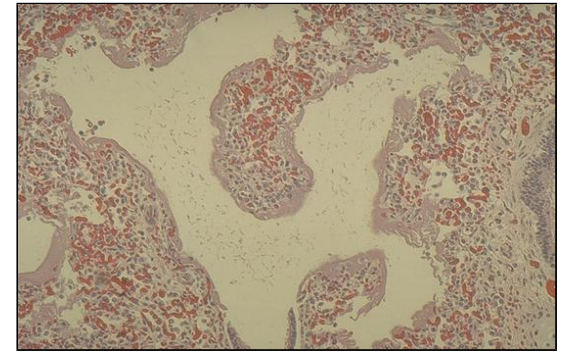

(a)

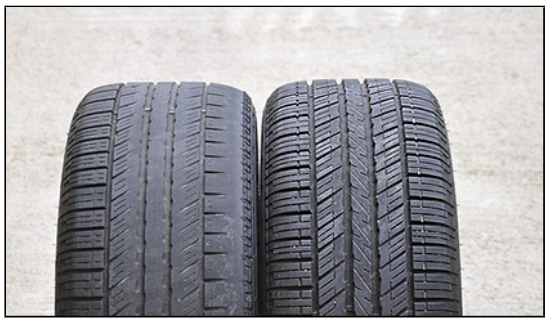

(c)

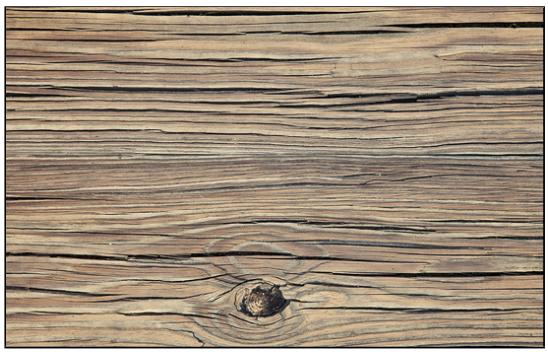

(e)

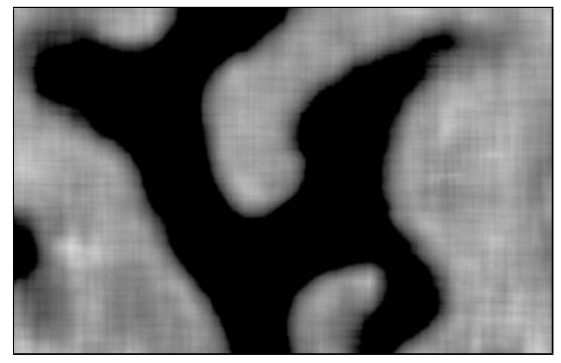

(b)

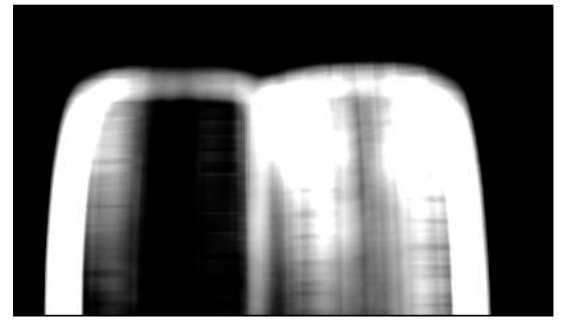

(d)

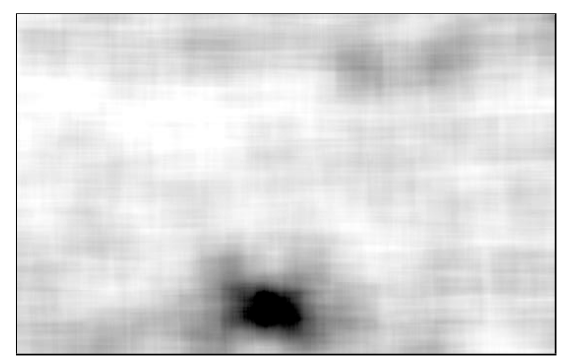

(f)

Figure 10: Experiments using the non-adaptive fuzzy models for pattern recognition. (a)(c)(e) Original images. (b)(d)(f) Membership degrees from (a)(b)(c) obtained by applying the fineness, contrast and directionality models, respectively 


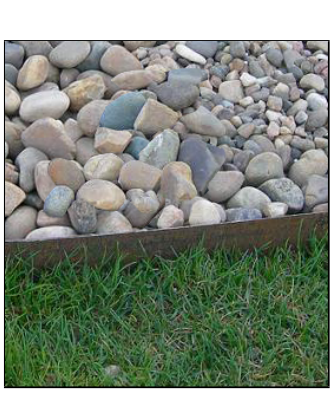

(a)

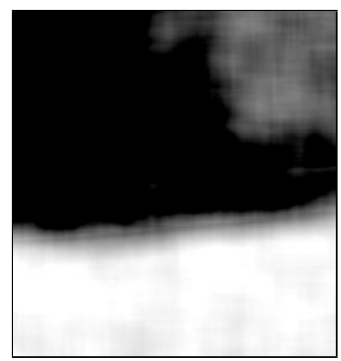

(d)

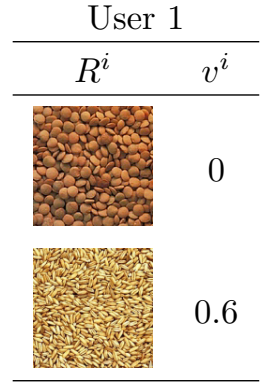

(b)

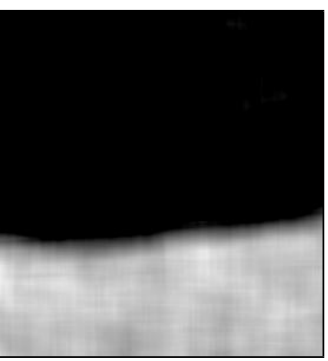

(e)

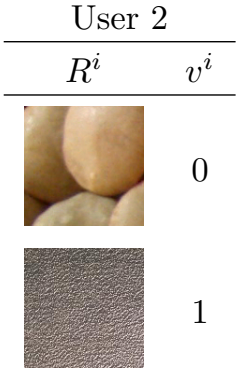

(c)

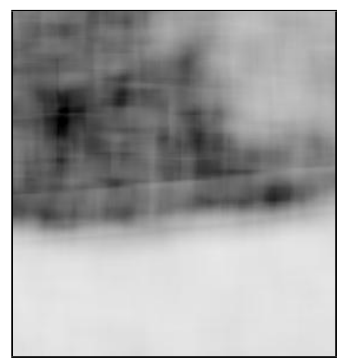

(f)

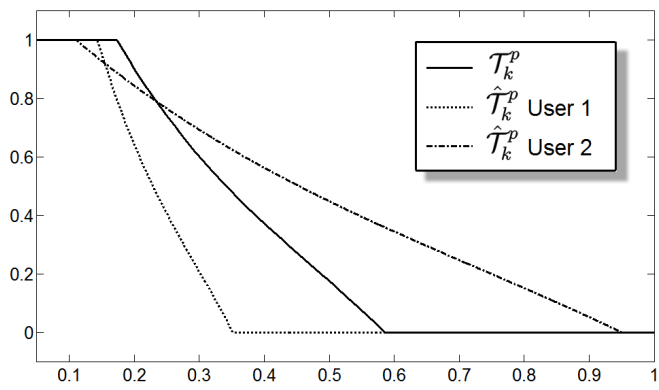

(g)

Figure 11: Adaptation of the fuzzy fineness model. (a) Original image. (d) Mapping from the original image to fineness values using the non-adaptive model. (b)(c) Samples representing the particular fineness perception of two different users. (e)(f) Mapping using the fuzzy model adapted according to each user's perception. (g) Membership function for the original and the adapted models. 
non-adaptive model. It can be noticed that three different degrees of fineness are shown: a coarse texture (pixels in black) corresponding to the big stones, a fine texture (pixels in white) corresponding to the grass, and a intermediate coarseness texture (pixels with an intermediate grey level) corresponding to gravel.

Let's adapt the non-adaptive model to the fineness perception of two hypothetical users. Figure 11(b) shows two texture images given by the user 1 to represent his particular fineness perception, and the perception degrees of fineness given by this user. It can be seen that the image $R^{1}$, that has an intermediate fineness perception according to the non-adaptive model (a fineness membership degree around 0.5 ), is now perceived as a coarse texture by this user $\left(v^{1}=0\right)$. The perception degree of fineness given by the user to the image $R^{2}\left(v^{2}=0.6\right)$ is also smaller than the fineness membership degree obtained with the non-adaptive model. The adapted membership function $\hat{\mathcal{T}}_{k}^{p}$ obtained on the basis of this information, together with the non-adaptive model $\mathcal{T}_{k}^{p}$, is shown in Figure $11(\mathrm{~g})^{11}$. Comparing both functions, it should be noticed that, according to the particular perception of the user 1, all the textures will be considered coarser than those obtained when $\mathcal{T}_{k}^{p}$ is applied. Figure 11(e) shows a mapping from the natural image in Figure 11(a) using $\hat{\mathcal{T}}_{k}^{p}$. It can be seen that in this case the gravel is also considered as a coarse texture, and the grass is not considered as fine as in Figure 11(d).

Figure 11(c) shows the samples given by a second user in order to represent his particular fineness perception. According to this user, a coarse texture corresponds to very big texture primitives, whereas a fine texture corresponds to very small texture primitives. This implies that the adapted membership function $\hat{\mathcal{T}}_{k}^{p}$ is obtained by expanding $\mathcal{T}_{k}^{p}$ in the domain of the measure, as it is shown in Figure 11(g). The mapping from the original image to its fineness values using $\hat{\mathcal{T}}_{k}^{p}$ is shown in Figure 11(f).

In the next two experiments, shown in figures 12 and 13, the proposed fuzzy sets for the contrast and directionality properties are adapted to the particular perception of different users. For the first one, we have considered the natural image shown in Figure 12(a), where several textures with different perception degrees of contrast are present. Figure 12(c) shows a mapping from the original image to contrast values using the proposed non-adaptive model. It can be noticed that, as it was expected, three different degrees of contrast are shown: a high contrasted texture (pixels in white) corresponding to the zebra, a low contrasted texture (pixels in black) corresponding to the sky, and a half-contrasted texture (pixels with an intermediate gray level) corresponding to the grass.

Let's adapt the non-adaptive model to the contrast perception of a hypothetical user. In this case, we suppose that the user gives only one sample image, shown in Figure 12(b), to represent his particular perception. Specifically, it contains the texture of the grass, that, as seen above, has an intermediate contrast perception according to the non-adaptive model. However, it is perceived

\footnotetext{
${ }^{11}$ For clarity in notation, $p$ is not replaced by the name of the property in $\mathcal{T}_{k}^{p}$ and $\hat{\mathcal{T}}_{k}^{p}$ (in this case $p=$ fineness $)$
} 


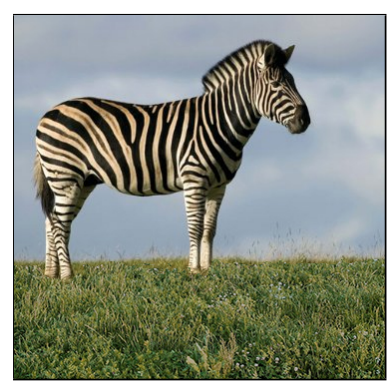

(a)

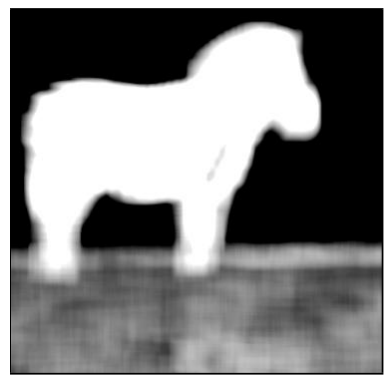

(c)

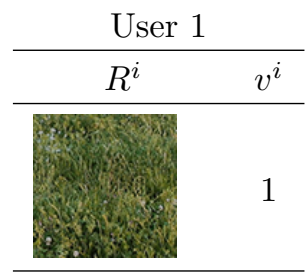

(b)

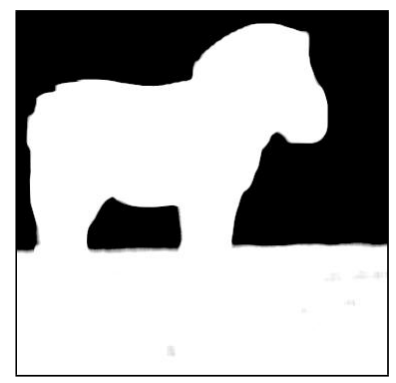

(d)

Figure 12: Adaptation of the fuzzy contrast model. (a) Original image. (b) Mapping from the original image to contrast values using the non-adaptive model. (c) Sample representing the particular contrast perception of an user. (d) Mapping using the fuzzy model adapted according to the user's perception.

as a very contrasted texture by this user $\left(v^{1}=1\right)$. Figure $12(\mathrm{~b})$ shows a mapping from the natural image using this adapted model. It can be noticed that the region of the image corresponding to the grass is considered as a very contrasted texture, as well as the zebra, matching the particular perception of this user.

For the next experiment, the natural image shown in Figure 13(a) has been considered, where several textures with different perception degrees of directionality are present. Figure 13(c) shows a mapping from the original image to directionality values using the proposed non-adaptive model. It can be noticed that this mapping represents the directionality of the different textures according to the average human perception: the pile of toothpicks has a high directional texture (pixels in white), the pile of lentils is considered as a low directional texture (pixels in black), while the curved pasta has an intermediate directionality.

As in the previous experiment, we suppose a hypothetical user that gives one sample image to represent his particular perception, shown in Figure 13(b). This image, that has an intermediate directionality perception according to the 


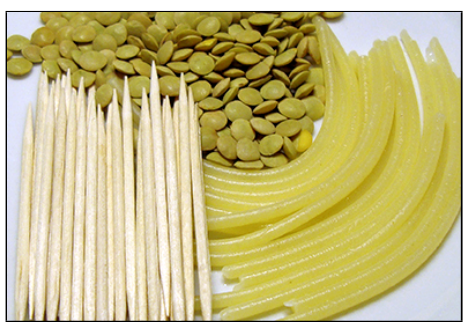

(a)

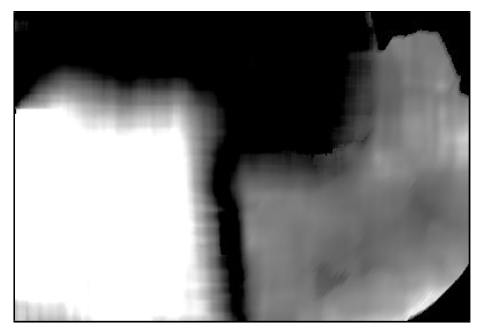

(c)

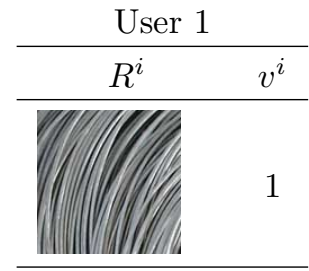

(b)

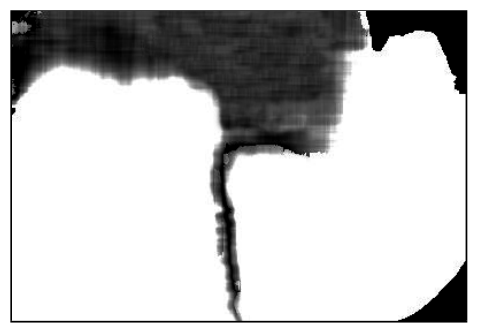

(d)

Figure 13: Adaptation of the fuzzy directionality model. (a) Original image. (b) Mapping from the original image to directionality values using the nonadaptive model. (c) Sample representing the particular directionality perception of an user. (d) Mapping using the fuzzy model adapted according to the user's perception.

non-adaptive model (similar to the curved pasta shown in the original image), is perceived as a very directional texture by this user $\left(v^{1}=1\right)$. Figure $13(\mathrm{~d})$ shows a mapping from the natural image using this adapted model. It can be seen that in this case the region of the pasta is also considered as a very directional texture, matching the particular perception of this user.

\subsection{Adaptation to image context}

Figure 14 presents an example where the fuzzy fineness model is adapted to the image context. Figure 14(a) shows an image that is composed by two textures with different fineness degrees. The mapping from this image to fineness values using the non-adaptive model is shown in Figure 14(b). It can be noticed that the texture within the lower left area is considered as very coarse according to the non-adaptive model, and the texture of the rest of the image is considered as fine. Figure 14(c) shows the mapping to the fineness values using the fuzzy model adapted to the image context. As commented in section 6.2, this adaptation is imposed by the inhibition present in the image, that depends on the coarsest and the finest texture in the image. It can be seen that this mapping is very 


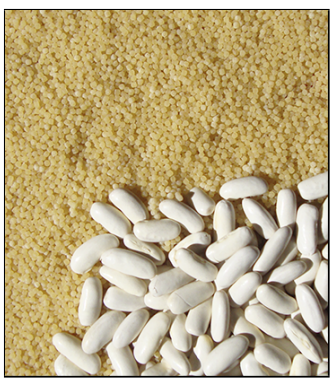

(a)

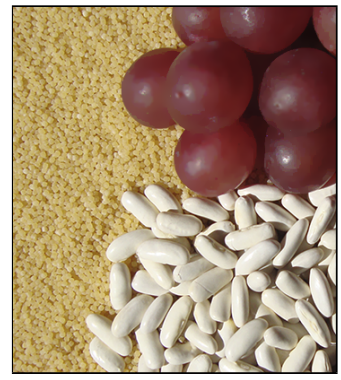

(d)

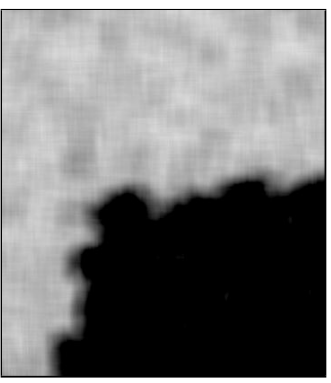

(b)

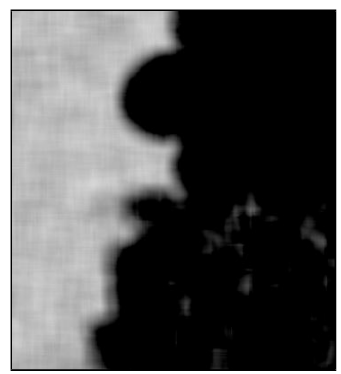

(e)

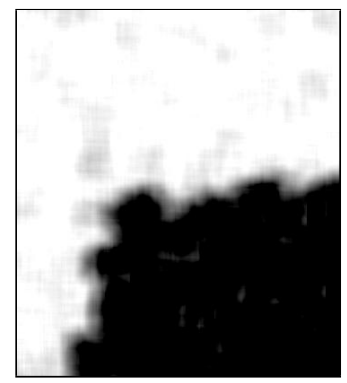

(c)

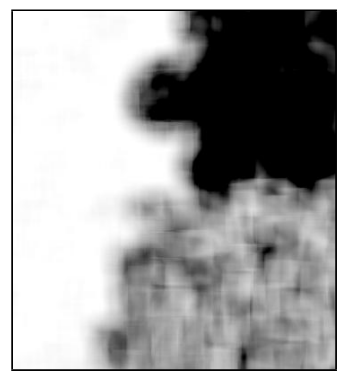

(f)

Figure 14: Adaptation of the fuzzy fineness model to the image context. (a)(d) Original images. (b)(e) Mapping from the original image to fineness values using the proposed non-adaptive model. (c)(f) Mapping using the fuzzy model adapted to the image context.

similar to Figure 14(b). The only difference is that the fine texture is considered finer than in Figure 14(b), due to the presence of the other texture, that is very coarse.

Figure 14(d) shows an image in which a new texture has been added to the image shown in Figure 14(a). The texture within the lower left area is the same as in Figure 14(a), but it may be perceived as finer by humans, because of the presence of the new texture, that is much coarser. This effect is more noticeable if the images are observed separately. Figure 14(e) shows the mapping to the fineness values using the non-adaptive model, that doesn't take into account the changes in the fineness perception due to the image context. Thus, the lower left area, as well as the new texture, are considered as very coarse, as in Figure 14(b). Figure 14(f) shows the mapping to the fineness values using the fuzzy model adapted to the image context. It can be noticed that in this case only the new texture is considered as very coarse. The texture within the lower left area has an intermediate fineness degree, which matches the human fineness perception influenced by the image context.

A similar experiment is shown in Figure 15, where the fuzzy contrast model 


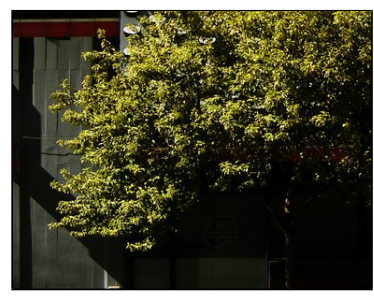

(a)

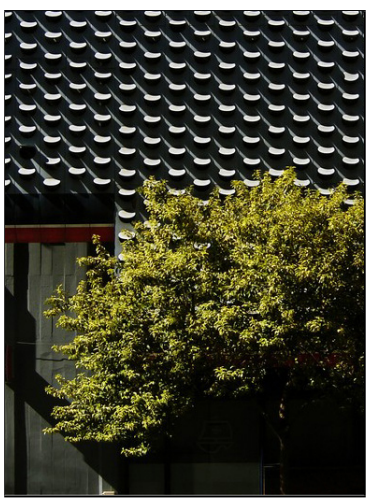

(d)

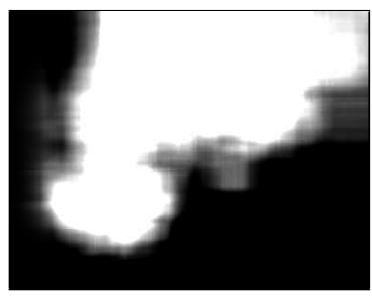

(b)

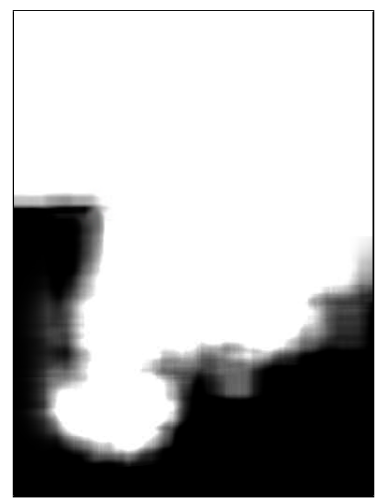

(e)

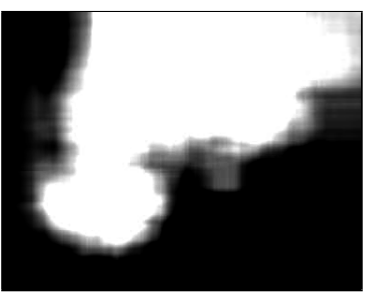

(c)

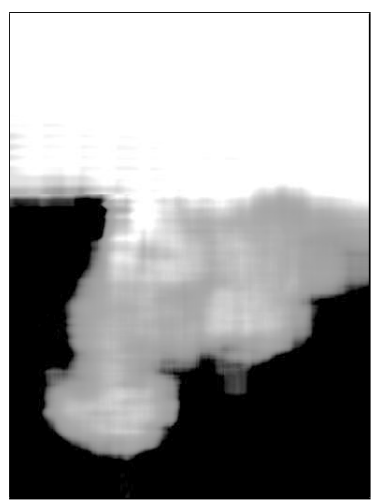

(f)

Figure 15: Adaptation of the fuzzy contrast model to the image context. (a)(d) Original images. (b)(e) Mapping from the original image to contrast values using the proposed non-adaptive model. (c)(f) Mapping using the fuzzy model adapted to the image context.

is adapted to the image context. Consider the natural image shown in Figure 15(a). The region corresponding to the tree leaves may be perceived as a very contrasted texture by humans (as it is shown in the mappings of figures 14(b) and 14(c)). Now consider the image shown in Figure 14(d). In this case the presence of a new texture, that is much more contrasted than the others, may inhibit the contrast perception. Figure 15(e) shows the mapping to the contrast values using the non-adaptive model. We can see that the region of tree leaves, as well as the new texture, is considered as very contrasted, as in Figure 15(b). Figure 15(f) shows the mapping to the contrast values using the fuzzy model adapted to the image context. It can be noticed that in this case only the new texture is considered as very contrasted. The texture corresponding to the tree leaves has an intermediate contrast degree, which matches the human contrast perception influenced by the image context. 


\section{Conclusions and future works}

In this paper, an adaptive fuzzy approach has been proposed to model perceptual properties of texture, taking into account the imprecision associated to them, as well as the subjectivity of the human perception. In particular, we have focused our study on the properties of coarseness, contrast and directionality. For this modelling, a methodology in two phases has been applied. In the first one, initial fuzzy sets (non-adaptive models) have been defined to model the average perception about texture properties. In our approach, some of the most representative measures in the literature have been employed as reference set, obtaining a group of fuzzy sets for each property. In order to analyze the ability of these fuzzy models to represent the corresponding property, goodness measures have been proposed taking into account the human assessments extracted from the poll. Thus, according to the ranking of measures shown in Table 1, we have concluded that the fuzzy models obtained by using the measure of Amadasun in the case of fineness, and the measures of Tamura for contrast and directionality are the most suitable one. The proposed non-adaptive models have been employed in several experiments, obtaining results that match the average human perception about each property, as has been shown in section 7.2. Moreover, the use of a unique fuzzy set for texture modelling has allowed its application to pattern recognition problems (e.g. the examples shown in Figure 10).

In the second phase of our approach, we have proposed a methodology to adapt the non-adaptive fuzzy sets in order to take into account the user's particular perception about the texture presence and the changes in perception influenced by the image context. Several experiments have been performed in order to analyze the ability of the adapted models obtained with the proposed methodology to represent different perceptions of the properties. In the experiments shown in section 7.3, fuzzy models adapted to particular users' profiles have been obtained, and we have seen that the perception degrees provided by these models match what the particular user would expect. Similar satisfactory results have been obtained in the experiments shown in section 7.4, where the fuzzy models have been adapted to the image context.

Regarding to the future work, as it was pointed out, in the fuzzy sets proposed in this paper only one crisp measure is used as reference set, which implies that only the ability of this measure to capture the corresponding property is considered in the modelling. However, it is natural to assume that the combination of different computational measures may improve the characterization of texture properties. In this sense, we are working on a methodology to define the non-adaptive fuzzy sets on the domain of a combination of several measures, and to adapt the corresponding n-dimensional models to user's profiles and the image context. In addition, we will extend the proposed methodology to other perceptual texture properties, like regularity or line-likeness. 


\section{Acknowledgements}

This work has been partially supported by the Spanish Government under the project TIN2014-58227 "Descripción linguística de información visual mediante técnicas de minería de datos y computación flexible". We also would like to thank Dr. Daniel Sánchez for his valuable assistance in the field of fuzzy logic, as well as Elena Galán-Perales for her contribution in the human assessment collection.

\section{References}

[1] J. C. Russ, The Image Processing Handbook, 3rd Edition, CRC Press and IEEE Press, 1999.

[2] A. R. Rao, G. L. Lohse, Identifying high level features of texture perception, Graphical Models and Image Processing 55 (3) (1993) 218-233.

[3] H. Tamura, S. Mori, T. Yamawaki, Textural features corresponding to visual perception, IEEE Transactions on Systems, Man and Cybernetics 8 (1978) 460-473.

[4] H. C. Lin, C. Y. Chiu, S. N. Yang, Finding textures by textual descriptions, visual examples, and relevance feedbacks, Pattern Recognition Letters 24 (14) (2003) 2255-2267.

[5] W. T. Lin, C. H. Lin, T. H. Wu, , Y. K. Chan, Image segmentation using the k-means algorithm for texture features, in: Proceedings of the International Conference on Computer, Electrical, and Systems Science, and Engineering, Venice, Italy, 2010, pp. 26-28.

[6] W. Niblack, R. Barber, W. Equitz, M. Flickner, E. H. Glasman, D. Petkovic, P. Yanker, C. Faloutsos, G. Taubin, The QBIC project: Querying images by content, using color, texture, and shape, in: Proceedings of Storage and Retrieval for Image and Video Databases, San Jose, CA, USA, 1993, pp. 173-187.

[7] S. Battiato, G. Gallo, S. Nicotra, Perceptive visual texture classification and retrieval, in: Proceedings of the 12th International Conference on Image Analysis and Processing, Washington, DC, USA, 2003, pp. 524-529.

[8] B. S. Manjunath, P. Salembier, T. Sikora (Eds.), Introduction to MPEG7, Multimedia Content Description Interface, John Wiley and Sons, Ltd., 2002.

[9] M. Nirmala, K. Karthikeyan, S. Appalabatla, R. A. Ahmed, Image interpretation based on similarity measures of visual content descriptors. An insight, International Journal of Computer Science and Emerging Technologies 2 (2) (2011) 242-248. 
[10] H. Aboulmagd, N. El-Gayar, H. Onsi, A new approach in content-based image retrieval using fuzzy, Telecommunication Systems 40 (1) (2009) 5566 .

[11] M. Flicker, H. Sawhney, W. Niblack, J. Ashley, Q. Huang, B. Dom, M. Gorkani, J. Hafner, D. Lee, D. Petkovic, D. Steele, P. Yanker, Query by image and video content: the QBIC system, IEEE Computer 28 (9) (1995) $23-32$.

[12] J. Han, K. K. Ma, Rotation-invariant and scale-invariant Gabor features for texture image retrieval, Image and Vision Computing 25 (9) (2007) $1474-1481$.

[13] A. K. Jain, F. Farrokhnia, Unsupervised texture segmentation using Gabor filters, Pattern Recognition 24 (12) (1991) 1167-1186.

[14] D. Zhang, A. Wong, M. Indrawan, G. Lu, Content-based image retrieval using Gabor texture features, in: Proceedings of the IEEE Pacific-Rim Conference on Multimedia, Sydney, Australia, 2000, pp. 392-395.

[15] S. Arivazhagan, L. Ganesan, Texture classification using wavelet transform, Pattern Recognition Letters 24 (9-10) (2003) 1513-1521.

[16] P. S. Hiremath, S. Shivashankar, Wavelet based features for texture classification, ICGST International Journal on Graphics, Vision and Image Processing 6 (3) (2006) 55-58.

[17] W. Y. Ma, B. S. Manjunath, A comparison of wavelet transform features for texture image annotation, in: Proceedings of the IEEE International Conference on Image Processing, Vol. 2, Washington, D.C., USA, 1995, pp. $256-259$.

[18] R. M. Haralick, Statistical and structural approaches to texture, Proceedings IEEE 67 (5) (1979) 786-804.

[19] M. Amadasun, R. King, Textural features corresponding to textural properties, IEEE Transactions on Systems, Man and Cybernetics 19 (5) (1989) $1264-1274$.

[20] N. Abbadeni, N. Ziou, D. S. Wang, Autocovariance-based perceptual textural features corresponding to human visual perception, in: Proceedings of the 15th International Conference on Pattern Recognition, Vol. 3, Barcelona, Spain, 2000, pp. 901-904.

[21] C. Y. Chiu, H. C. Lin, S. N. Yang, A fuzzy logic CBIR system, in: Proceedings of the 12th IEEE International Conference on Fuzzy Systems, Vol. 2, St Louis, MO, USA, 2003, pp. 1171-1176.

[22] S. Kulkarni, B. Verma, Fuzzy logic based texture queries for CBIR, in: Proceedings of the 5th International Conference on Computational Intelligence and Multimedia Applications, Xi'an, China, 2003, pp. 223-228. 
[23] B. Verma, S. Kulkarni, A fuzzy-neural approach for interpretation and fusion of colour and texture features for CBIR systems, Applied Soft Computing 5 (1) (2004) 119-130.

[24] J. Chamorro-Martínez, P. Martínez-Jiménez, A comparative study of texture coarseness measures, in: Proceedings of the 16th IEEE International Conference on Image Processing, Cairo, Egypt, 2009, pp. 1337-1340.

[25] J. Chamorro-Martínez, E. Galán-Perales, J. M. Soto-Hidalgo, Fuzzy sets for modelling fineness perception in texture images, in: Proceedings of the 2007 IEEE International Conference on Fuzzy Systems, London, 2007, pp. 1903-1908.

[26] J. Chamorro-Martínez, P. Martínez-Jiménez, J. M. Soto-Hidalgo, Defining bidimensional fuzzy sets for coarseness modelling in texture images, in: Proceedings of the 2009 IEEE International Conference on Fuzzy Systems, Jeju Island, Korea, 2009, pp. 1358-1363.

[27] B. B. Mandelbrot, The fractal geometry of nature, Freeman, San Francisco, 1982.

[28] S. Peleg, J. Naor, R. Hartley, D. Avnir, Multiple resolution texture analysis and classification, IEEE Transactions on Pattern Analysis and Machine Intelligence 6 (4) (1984) 518-523.

[29] M. M. Galloway, Texture analysis using gray level run lengths, Computer Graphics and Image Processing 4 (1975) 172-179.

[30] C. Sun, W. G. Wee, Neighboring gray level dependence matrix for texture classification, Computer Vision, Graphics and Image Processing 23 (1983) 341-352.

[31] S. I. Kim, K. C. Choi, D. S. Lee, Texture classification using run difference matrix, in: Proceedings of the 1991 IEEE Ultrasonics Symposium, Vol. 2, Orlando, FL, USA, 1991, pp. 1097-1100.

[32] J. S. Weszka, C. R. Dyer, A. Rosenfeld, A comparative study of texture measures for terrain classification, IEEE Transactions on Systems, Man, and Cybernetics 6 (4) (1976) 269-285.

[33] S. D. Newsam, C. Kammath, Retrieval using texture features in high resolution multi-spectral satellite imagery, in: Proceedings of Data Mining and Knowledge Discovery: Theory, Tools, and Technology VI, Vol. 5433, Orlando, FL, USA, 2004, pp. 21-32.

[34] H. Yoshida, D. D. Casalino, B. Keserci, A. Coskun, O. Ozturk, A. Savranlar, Wavelet-packet-based texture analysis for differentiation between benign and malignant liver tumours in ultrasound images, Physics in Medicine and Biology 48 (2003) 3735-3753. 
[35] P. Brodatz, Textures: a photographic album for artists and designers, Dover Publishing Co., 1966.

[36] R. R. Yager, On ordered weighted averaging aggregation operators in multicriteria decisionmaking, IEEE Transactions on Systems, Man and Cybernetics 18 (1) (1988) 183-190.

[37] A. E. Beaton, J. W. Tukey, The fitting of power series, meaning polynomials, illustrated on band-spectroscopic data, Technometrics 16 (1974) $147-185$.

[38] M. G. Kendall, A New Measure of Rank Correlation, Biometrika 30 (1/2) (1938) 81-93.

[39] C. Spearman, The proof and measurement of association between two things, American Journal of Psychology 15 (1904) 72-101.

[40] R. A. Fisher, Statistical Methods For Research Workers, Cosmo study guides, Cosmo Publications, 1925.

[41] S. S. Tripathy, R. Shekhar, R. S. Kumar, Texture retrieval system using intuitionistic fuzzy set theory, in: Proceedings of the 2011 International Conference on Devices and Communications, Jharkhand, India, 2011, pp. $1-5$. 